\title{
METAL TRANSPORT TO THE GASEOUS OUTSKIRTS OF GALAXIES
}

\author{
J. K. Werk ${ }^{1,2,4}$, M. E. Putman ${ }^{2}$, G. R. Meurer ${ }^{3}$, and N. Santiago-Figueroa ${ }^{2,5}$ \\ ${ }^{1}$ Department of Astronomy, University of Michigan, 500 Church St., Ann Arbor, MI 48109, USA; jwerk@ ucolick.org \\ ${ }^{2}$ Department of Astronomy, Columbia University, 550 West 120th Street, New York, NY 10027, USA \\ ${ }^{3}$ ICRAR/The University of Western Australia, 35 Stirling Highway, Crawley, WA 6009, Australia \\ Received 2010 September 24; accepted 2011 April 18; published 2011 June 17
}

\begin{abstract}
We present a search for outlying $\mathrm{H}$ II regions in the extended gaseous outskirts of nearby ( $D<40 \mathrm{Mpc}$ ) galaxies and subsequent multi-slit spectroscopy used to obtain the H II region nebular oxygen abundances. The galaxies in our sample have extended $\mathrm{H}$ I disks and/or interaction-related $\mathrm{H}$ I features that extend well beyond their primary stellar components. We report oxygen abundance gradients out to 2.5 times the optical radius for these galaxies which span a range of morphologies and masses. We analyze the underlying stellar and neutral $\mathrm{H}$ i gas distributions in the vicinity of the $\mathrm{H}$ II regions to understand the physical processes that give rise to the observed metal distributions in galaxies. These measurements, for the first time, convincingly show flat abundance distributions out to large radii in a wide variety of systems and have broad implications for galaxy chemodynamical evolution.
\end{abstract}

Key words: galaxies: abundances - galaxies: evolution - H II regions - ISM: kinematics and dynamics

\section{INTRODUCTION}

Through nuclear fusion and well-studied stellar evolution processes, stars with $M_{*}>8 \mathcal{M}_{\odot}$ generate metals in their cores and return them to the local interstellar medium (ISM) near the end of their lifetimes. Since galaxies are neither static nor isolated, these metals do not simply stay put. Instead, they disperse throughout the galaxy and sometimes flow into and out of the galaxy via galactic winds and galaxy interactions (Oey 2003; Tremonti et al. 2007; Oppenheimer \& Davé 2009).

The transport mechanisms that govern how the metals are distributed throughout and/or ejected from galaxies are widely debated and remain poorly understood. At the heart of the matter is the myriad of gas-phase metal distributions determined from observational data. Metals are evenly distributed in some spiral galaxies, while others exhibit steeply declining radial abundance gradients (Vila-Costas \& Edmunds 1992; Oey \& Kennicutt 1993). By comparison, dwarf galaxies tend to show shallow, if not flat, abundance gradients (Croxall et al. 2009). Very recent work by Kewley et al. (2010) indicates that close galaxy pairs also tend to have flatter metallicity gradients than those observed in typical isolated spiral galaxies. While there is a steep inner-galaxy abundance gradient in M83, there is an observed flattening of the radial abundance gradient at large radii ( $r>R_{25}$; Bresolin et al. 2009). It is most likely a combination of astrophysical processes that leads to the observed metal distributions in galaxies of various properties. For example, disk "viscosity" and metal mixing (Zaritsky 1992; Ferguson \& Clarke 2001), a radial dependence of star-forming activity (Phillipps \& Edmunds 1991), large-scale metal blowout (Tremonti et al. 2004), and merger-induced gas flows (Rupke et al. 2010) have all been put forth as potential causes for the observed metal distributions in star-forming, dwarf, and/ or interacting galaxies.

Characterizing the metal distribution in galaxies of all morphological types and masses over a wide range of galactocentric

\footnotetext{
4 Current address: Department of Astronomy and Astrophysics \& UCO/Lick Observatory, University of California, 1156 High Street, Santa Cruz, CA 95064, USA.

5 Current address: Department of Physics, Fisk University, Nashville, TN 37208, USA.
}

distances is essential for determining the relative importance of various metal-mixing processes. These processes, in turn, have significant bearing on our understanding of galaxy evolution, galaxy feedback, and star formation (SF). With regard to observational studies of galaxy-wide metallicity gradients, there are two key environments that have yet to be fully investigated: interacting or disturbed galaxies, and the far outer reaches of galaxies. It is in their gaseous outskirts that galaxies may reveal how they build themselves up over cosmic time, through interactions and gas flows. In this paper, we probe the chemical abundances of the far outer regions of disturbed and/or extended-gas, gas-rich galaxies, and therefore extend the study of metallicity distributions in galaxies to a new and important locale. To do this study, we use Hir regions that span a wide range of galactocentric distances: within the optical extent $\left(r<R_{25}\right)$ and beyond $\left(r>R_{25}\right.$, referred to as "outlying $\mathrm{H}_{\text {II }}$ regions"; Werk et al. 2010b). We search for these H II regions in narrowband images of galaxies in the Hi Rogues catalog (Hibbard et al. 2001).

The Hi Rogues catalog consists of 189 Hi synthesis maps of nearby galaxies with extended, unusual, or disturbed $\mathrm{HI}$ morphologies, including galaxies with extended (many times the optical extent) or warped $\mathrm{HI}$ distributions, interacting groups, minor and major mergers, intergalactic debris with no optical counterparts, and early-type galaxies with $\mathrm{HI}$ inside and/or outside their optical bodies. As the host galaxies of outlying $\mathrm{H}$ II regions are strongly correlated with disturbances, companions, extended gas, and interactions, this collection of "Rogue" galaxies provides a potentially rich hunting ground for outlying, massive SF (Werk et al. 2010b). Another benefit of the $\mathrm{H}$ I Rogues sample is that all galaxies have readily available $\mathrm{H}$ I synthesis maps, providing crucial information about the gaseous environments of the outlying stars. In these H I Rogue systems, the oxygen abundances of the extended and/or stripped outer gaseous material can place much-needed constraints on metal transport in gas-rich galaxies.

Extragalactic $\mathrm{H}_{\text {II }}$ regions have long been used as reliable indicators of the current metal abundance of the gas in their vicinity. At the low electron densities typical of $\mathrm{H}$ II regions $\left(n_{e}<10^{4} \mathrm{~cm}^{-3}\right)$, forbidden emission lines, such as [O III] $\lambda \lambda 4959,5007$ and $\left[\mathrm{O}_{\mathrm{II}}\right] \lambda \lambda 3727$, are characteristic features 
of $\mathrm{H}$ II region emission-line spectra, along with the radiative recombination lines of the hydrogen Balmer and Paschen series (e.g., $\mathrm{H} \alpha$ ). Because the strength of every emission line from every element present in the $\mathrm{H}$ II region depends on its chemical abundance in the nascent ISM gas, H II regions are valuable metallicity indicators (Osterbrock 1989). As such, they trace the SF history of the gas and act as chemical tags that can aid in determining the origin of the gas.

The practice of determining chemical abundance gradients using $\mathrm{H}$ II region emission-line spectra was initiated by Searle (1971), who inferred a declining Galactic O/H abundance gradient from the observed increase in $\mathrm{H}$ II region excitation and temperature with radius (with [O III] $\lambda 4959, \lambda 5007 /[\mathrm{O}$ II $] \lambda 3727$ and $[\mathrm{O} \mathrm{III}] / \mathrm{H} \beta$ ratios). Shields \& Tinsley (1976) confirmed the anti-correlation between $T_{\text {eff }}$ and metallicity and attributed the radial gradients to a metallicity-dependent upper mass limit for SF such that $M_{\text {upper }} \propto Z^{-1 / 2}$, a scaling theoretically determined by Kahn (1974) who modeled massive SF with a radiation "cocoon." Since then, numerous detailed studies have confirmed the overall trend for the decrease in oxygen abundance with galactocentric radii (e.g., Oey \& Kennicutt 1993; Zaritsky et al. 1994; Bresolin et al. 1999; Kennicutt et al. 2003) in a large number of spiral (barred + unbarred) and irregular star-forming galaxies, although the underlying physical cause is still a subject of debate. The work of Zaritsky et al. (1994) finds the radial oxygen abundance gradients of nearby spiral galaxies to range from $-0.03 \mathrm{dex} / \mathrm{kpc}^{-1}$ to $-0.15 \mathrm{dex} / \mathrm{kpc}^{-1}$.

Thus far, only a handful of measurements of gas-phase abundances at large optical radii $\left(r>R_{25}\right)$ have emerged (Ferguson et al. 1998; Bresolin et al. 2009), and many questions remain about the metal content in the outermost regions of galaxies. Our study aims to address those questions by determining the oxygen abundances of numerous inner and outlying $\mathrm{H}$ II regions in $13 \mathrm{H} \mathrm{I} \mathrm{Rogue} \mathrm{galaxies.} \mathrm{The} \mathrm{morphologically} \mathrm{diverse} \mathrm{nature}$ of our sample of galaxies allows us to consider the role played by galaxy interactions in the observed metal distributions.

This paper proceeds as follows: Section 2 describes imaging and spectroscopic data obtained at various telescopes over the course of several years; Section 4 reviews our methods for obtaining strong-line oxygen abundances from $\mathrm{H}$ II region emission-line spectra; Section 5 presents radial oxygen abundance gradients reaching beyond $R_{25}$ for $13 \mathrm{H}$ I Rogues with outlying $\mathrm{H}$ II regions; Section 6 discusses these results and comments on the physical mechanisms that could be responsible for the metal distributions; and Section 7 summarizes and concludes. Our Gemini multi-slit data allow the largest to-date observational study of the metal content of outlying gas (beyond $R_{25}$ ), and how it compares to the metal content of gas within the galaxy.

\section{OPTICAL IMAGING AND H I DATA}

\subsection{Ha Imaging}

In 2005 April, over the course of four clear nights, we imaged 27 galaxies from the H I Rogues catalog (Hibbard et al. 2001) in $\mathrm{H} \alpha$ and $R$-band continuum with the MDM $2.4 \mathrm{~m}$ Hiltner telescope. The MDM $2.4 \mathrm{~m}$ telescope direct images, with the Echelle CCD, have a field of view of $9.5 \times 9.5$ and a plate scale of 0.275 pixel $^{-1}$. The average seeing over the course of these four nights was $1^{\prime \prime} .2$. These observations were made in a similar fashion to the $\mathrm{H} \alpha$ imaging of the Survey of Ionization in Neutral Gas Galaxy (SINGG) survey (Meurer et al. 2006). We used four of the SINGG narrowband filters with bandpasses that corresponded to the $\mathrm{H} \alpha$ emission line at the target galaxy's velocity. The central wavelengths, in angstroms, of the filters used are 6568, 6596, 6605, and 6628, each with an FWHM of $\sim 30 \AA$ (Meurer et al. 2006) contains a full description of the SINGG filters). Exposure times were $3 \times 600 \mathrm{~s}$ in $\mathrm{H} \alpha$ and $3 \times 120 \mathrm{~s}$ in $R$. Targets were selected from the full H i Rogues catalog on the basis of their visibility from MDM observatory and their recessional velocities, such that the redshifted $\mathrm{H} \alpha$ emission line is encompassed by the SINGG $\mathrm{H} \alpha$ filters' narrow bandpasses. Galaxies were also selected to have angular optical and H i extents such that they were contained in the 9'.5 field of view. Observations of several different spectrophotometric standards were used to flux-calibrate the data.

We performed a basic reduction of the data using IRAF CCD processing routines for bias-subtraction and flat-field division. Images were then combined and astrometry was performed using the SINGG IDL pipeline routines (see Meurer et al. 2006) modified specifically for the MDM $2.4 \mathrm{~m}$ telescope. Photometry was performed on the images based on observations of spectrophotometric standards calibrated to the $\mathrm{AB}$ magnitude system. We followed the basic SINGG procedure detailed in Appendix A of Meurer et al. (2006). The average 5 $\sigma$ limiting $\mathrm{H} \alpha$ flux for a point source is $\sim 1 \times 10^{-16} \mathrm{erg} \mathrm{cm}^{-2} \mathrm{~s}^{-1}$ and the average $5 \sigma$ limiting $R$-band magnitude is $\sim 24$.

\subsection{Searching Images for Outlying H II Regions}

We searched the reduced, calibrated MDM H $\alpha$ and $R$-band images for ELdots (emission-line dots; see Werk et al. 2010b) using the basic selection criteria outlined in Werk et al. (2010b), with the exception of the requirement that the ELdot lie beyond $2 \times R_{25}$. For reference, ELdots are defined to be strong emission-line point sources in narrowband images that lie well outside the broadband optical emission of nearby galaxies (Werk et al. 2010b). Generally, ELdots can be outlying $\mathrm{H} \alpha$ emitting $\mathrm{H}$ II regions at a similar velocity to the target galaxy or they can be background galaxies emitting a different line ([O III $] \lambda 5007$, [O II] $\lambda \lambda 3727$, or $\mathrm{H} \beta$ ) that is redshifted into the narrow filter passband used for the observations. For this study, we selected by-eye sources that appeared to lie beyond the main $R$-band optical extents of the potential host galaxies. The reason for relaxing our projected distance requirement is that $R_{25}$ is not well defined for merging, interacting, and/or irregular, asymmetric galaxies. For comparison to the sample in Werk et al. (2010b), and an estimate of the ELdots' projected distances from the main optical extents of their host galaxies, we did calculate an approximate $R_{25}$ at a later time. In order to obtain the approximate location of $R_{25}$ in these messy systems, we used the IRAF task ELLIPSE found in the STSDAS package, fitting elliptical isophotes to the target Rogue galaxies in the $2.4 \mathrm{~m}$ MDM $R$-band images. In one case (NGC 3227), two merging galaxies had to be fit with a single ellipse. In other cases, incidences of tails, plumes, and warps caused the fixed-center elliptical isophotes to be significantly larger than they otherwise would have been. The laxity of the $R_{25}$ fitting should be noted, and the quantity itself should be treated as an optical radial reference point when discussing oxygen abundance gradients. All of the Rogue ELdots, originally identified by eye, lie beyond this optical radius.

Thirteen of the $27 \mathrm{HI}$ Rogues imaged with the MDM $2.4 \mathrm{~m}$ were found to have over 40 ELdots in total. We began the process of spectroscopically confirming their association with the target galaxy, with longslit spectroscopy at the MDM $2.4 \mathrm{~m}$ telescope (Section 3.2). Various difficulties with blind offsets 


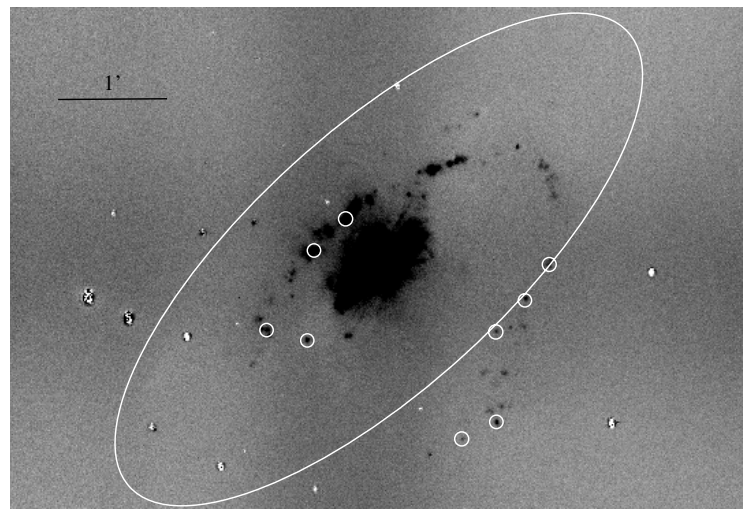

(a) NGC 2146

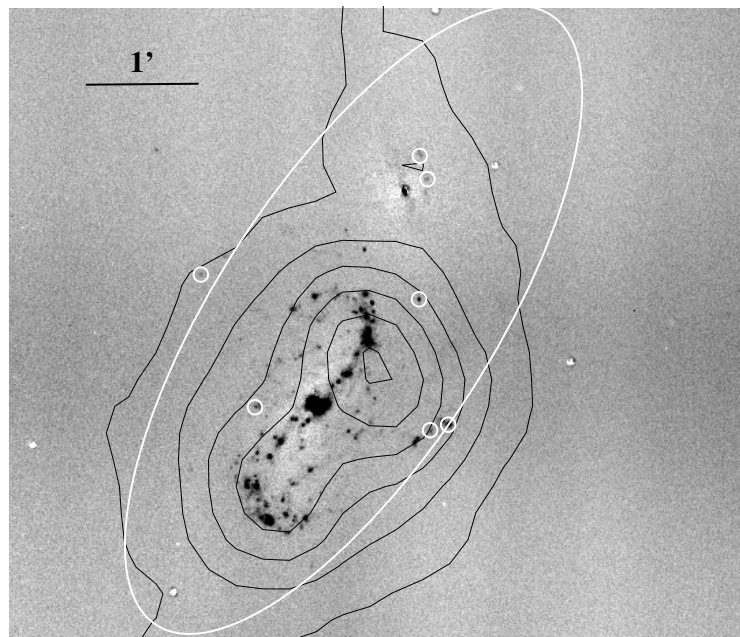

(c) NGC 3227 ; with $\mathrm{HI}$ contours at 1.5 to $8.5 \mathrm{M}_{\odot}$ $\mathrm{pc}^{-2}$

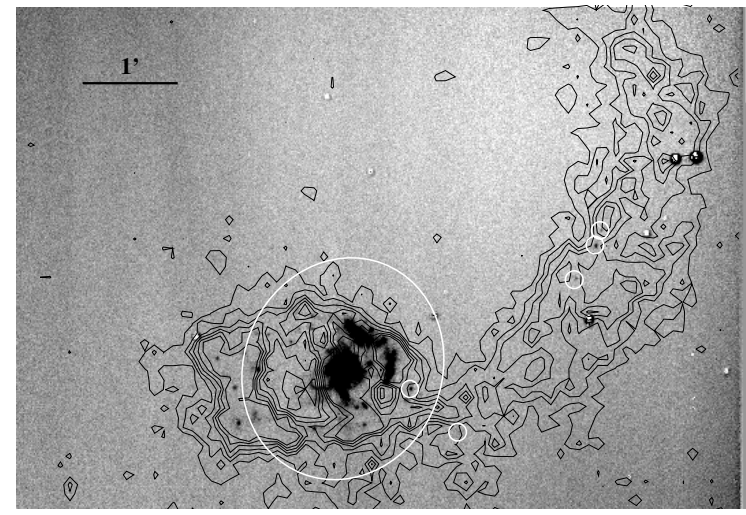

(b) NGC 2782; with $\mathrm{HI}$ contours at 2.5 to $10.5 \mathrm{M}_{\odot}$ $\mathrm{pc}^{-2}$

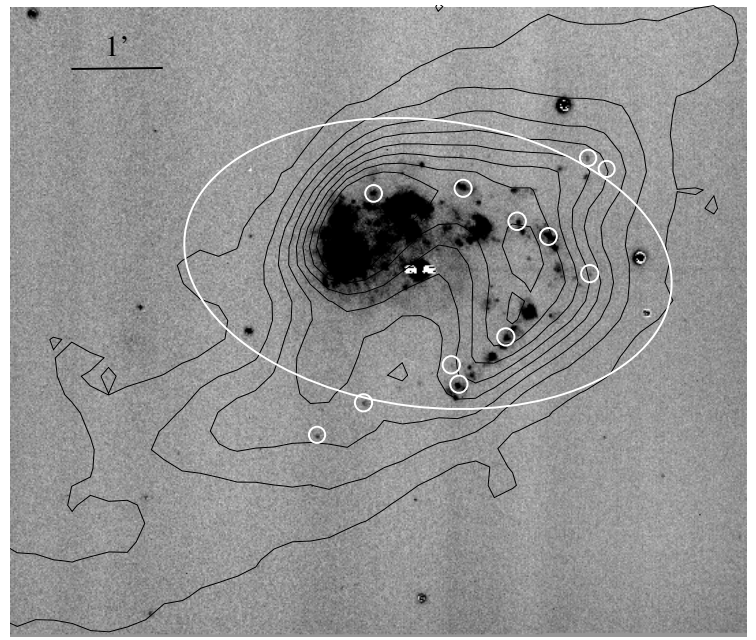

(d) NGC 3239 ; with $\mathrm{HI}$ contours at 0.5 to $8.5 \mathrm{M}_{\odot}$ $\mathrm{pc}^{-2}$

Figure 1. Continuum-subtracted $\mathrm{H} \alpha$ emission images from the MDM $2.4 \mathrm{~m}$ telescope, as described in Section 2, overlaid with H I column density contours, where available. Original references and additional details on the $\mathrm{H}$ I data (beam size, etc.) can be found in the online H I Rogues catalog at http://www.nrao.edu/astrores/HIrogues/ and in Section 5. The white ellipse shows the location of the elliptical $R$-band 25th magnitude isophote $\left(R_{25}\right)$ for the galaxy, while the smaller white circles point to the locations of the H II regions for which we obtained GMOS-N multi-slit spectra. Images are aligned to a world-coordinate system such that north is upward, and east is to the left.

and large observational overhead forced us to discontinue the follow-up longslit spectroscopy before it was complete. Yet, of the dozen ELdot spectra that were obtained, all were confirmed as outlying $\mathrm{H}$ II regions (the $\mathrm{H} \alpha$ emission line, instead of any of the redshifted strong blue emission lines, falls in the SINGG narrow bandpass). This high confirmation rate led us to pursue deeper multi-slit spectroscopy with the Gemini $8 \mathrm{~m}$ telescope, as described in Section 3.1.

\subsection{Properties of the Sample of 13 H I Rogues}

Figure 1 shows the $\mathrm{H} \alpha$ continuum-subtracted images for the $13 \mathrm{H}$ I Rogue fields in which we found ELdots. For nine of these galaxies, we also show H I column density contours generated from reduced $\mathrm{H}$ I data cubes kindly provided by several project PIs (see Table 1). These images highlight the inner- and outergalaxy SF and often do not show the full MDM field of view or the full extent of the H I distribution. We show $R_{25}$ on the images, in addition to all of the $\mathrm{H}$ II regions (outer + inner) for which we were able to obtain GMOS multi-slit spectra (see the next section). Regarding the few galaxies for which $\mathrm{HI}$ data cubes were not available to us, we refer the reader to the online H I Rogues catalog and references therein (Hibbard et al. 2001). Table 1 lists the optical, H I, and derived properties of this sample. Column 7 provides an estimate of the total oxygen abundance for each galaxy from the literature. In most cases, the oxygen abundance measurement was made with various strong emission-line ratios for H II regions in the central parts (within $R_{25}$ in all cases) of each galaxy. Column 8 lists the Hi morphology code from the Hi Rogues catalog for each target. The codes are defined in the caption of Table 1 , and the H I morphology of each target is described in greater detail in Section 5.

For the calculations we perform in Section 6, we derive the total baryonic (stellar + gas) and global star formation rate (SFR) for each galaxy in our sample. To obtain a value of the stellar mass, we use the published total integrated broadband magnitudes in $B$ - and $V$-bands available on NED in conjunction with the stellar mass-luminosity/color relation from Bell et al. (2003). Most of the total $B$-band and $V$-band apparent magnitude estimates come from the 1991 De Vaucoleurs Catalog, using a combination of new and previously published data, and are corrected for internal and Milky Way extinction. UGC 5288 is an exception in this regard (not included in the De Vaucoleurs 


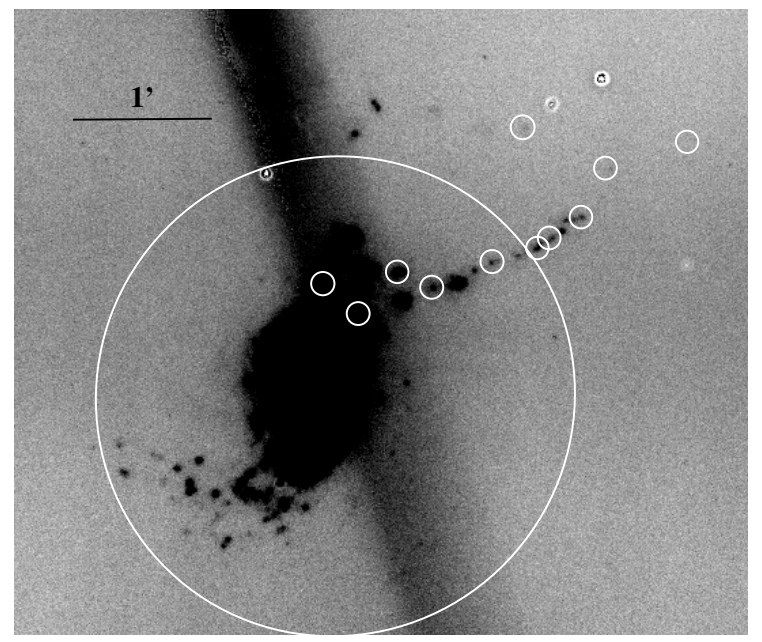

(e) NGC 3310

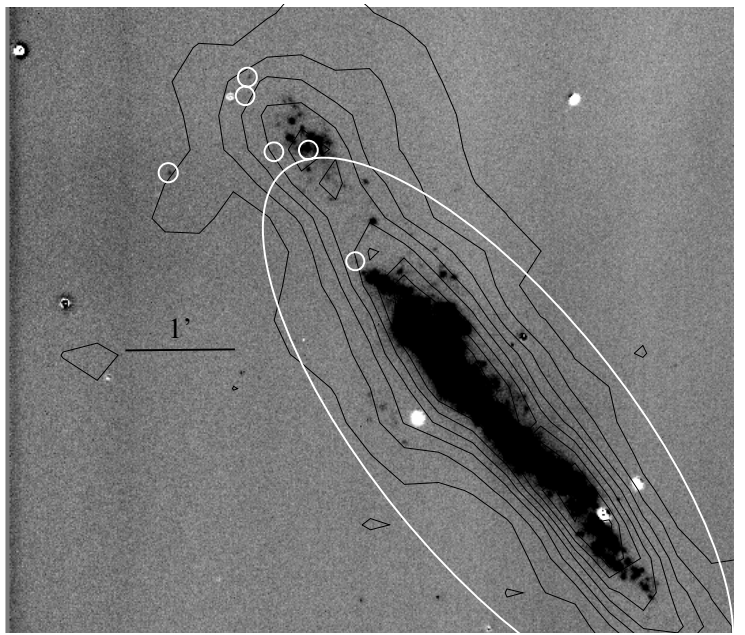

(g) NGC 3432; with $\mathrm{HI}$ contours at 6.5 to $60 \mathrm{M}_{\odot}$ $\mathrm{pc}^{-2}$

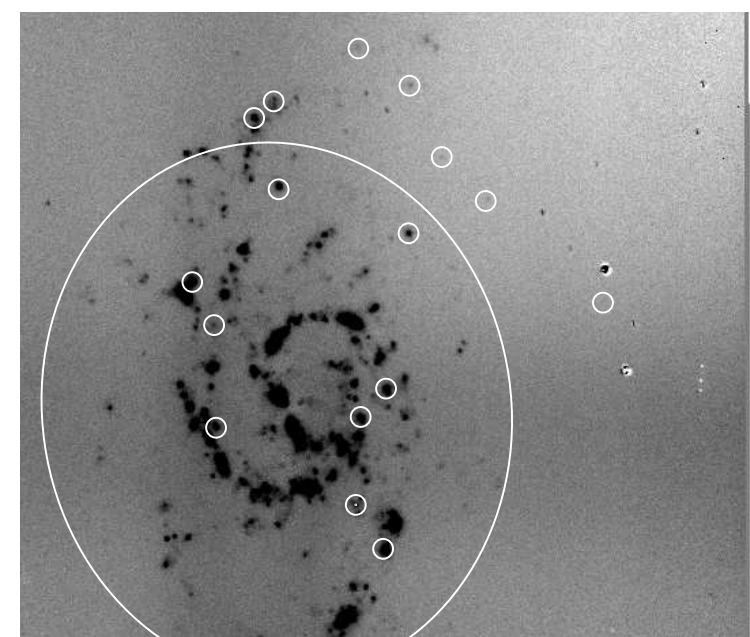

(f) NGC 3359

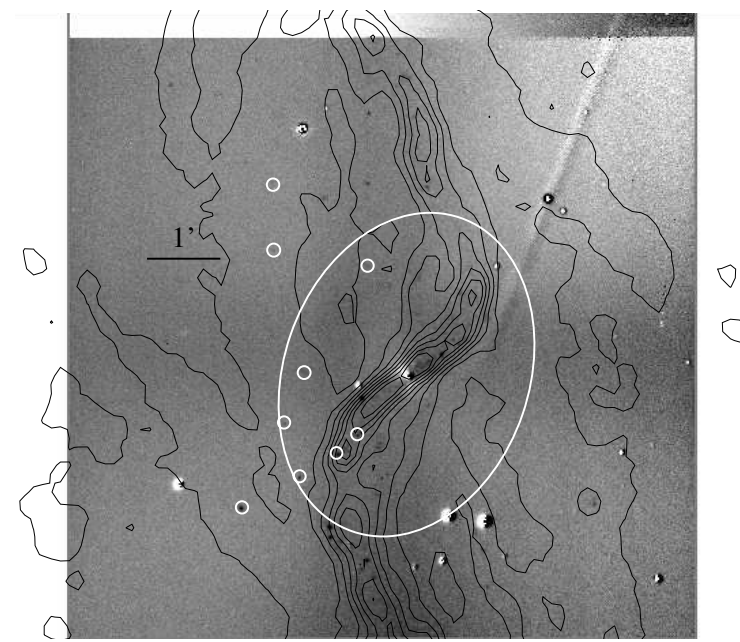

(h) NGC 3718; with $\mathrm{HI}$ contours at 0.5 to $20 \mathrm{M}_{\odot}$ $\mathrm{pc}^{-2}$

Figure 1. (Continued)

Catalog), and therefore we used the broadband optical $B$ - and $R$-band photometry from van Zee (2000). To obtain the total stellar mass from the $B$-band luminosity and the $B-V$ color (sometimes $B-R$ ), we use Table 7 of Bell et al. (2003) which gives coefficients for their derived stellar mass-luminosity/color relation for Sloan Digital Sky Survey (SDSS) galaxies. These stellar masses are accurate to within 50\% and are given in Column 11 of Table 1 . In some cases, we are able to compare our $B-V$ results with other calibrations of the Bell et al. (2003) mass-luminosity/color relations using different broadband filters, which give values that are consistent within the errors. The H I masses in Column 12 of Table 1 are obtained from the literature, and references are given in Column 10.

We calculate SFRs using the relation SFR $\left(\mathcal{M}_{\odot} \mathrm{yr}^{-1}\right)=$ $5.3 \times 10^{-42} L(\mathrm{H} \alpha)\left(\mathrm{erg} \mathrm{s}^{-1}\right)($ Calzetti 2008) and obtain total $\mathrm{H} \alpha$ luminosities from the MDM $2.4 \mathrm{~m}$ images within an elliptical aperture at $2 \times R_{25}$. These total $\mathrm{H} \alpha$ luminosities are corrected for Galactic extinction (Schlegel et al. 1998) and for the contribution of $[\mathrm{N}$ II] $\lambda 6583$ using the $[\mathrm{N}$ II] $/ \mathrm{H} \alpha$ emissionline ratios from the literature (listed in Column 9 of Table 1) and convolving this contamination with the SINGG narrowband filter transmission curve (see Appendix A of Meurer et al. 2006 for details). This $[\mathrm{N} \mathrm{II}] / \mathrm{H} \alpha$ emission-line ratio for each galaxy represents a global average (from integrated spectra or averages of individual $\mathrm{H}$ II regions in the galaxy) over its optical extent. The only exception is NGC 3227, in which only one measurement of an $\mathrm{H}_{\text {II }}$ region at $\sim 0.8 \times$ $R_{25}$ was available. We did not attempt to add in the potential effects from metallicity gradients across the galaxy since such effects would be negligible in light of our rather high photometric errors. Considering errors in the continuum subtraction, occasional $\mathrm{H} \alpha$ filter reflections/diffuse patterns, and uncertainties in the extinction correction, we estimate that these total $\mathrm{H} \alpha$ luminosities, and thus the total SFRs, are accurate to within $\sim 20 \%$.

Table 2 lists the remaining 14 targets in which we did not find any ELdots, along with their $\mathrm{H}$ I morphologies, distance, and $R_{25}$. Although their properties are not fundamentally different from those of the H I Rogues with ELdots, we do note that the only three optical early-type elliptical galaxies with $\mathrm{H}$ I that we imaged with the MDM $2.4 \mathrm{~m}$ appear in this sample.

\subsection{Comparison with an Unbiased Sample of H I-selected Galaxies}

The H I Rogues sample is neither complete nor representative. It was compiled as a sample largely dominated by interacting or 


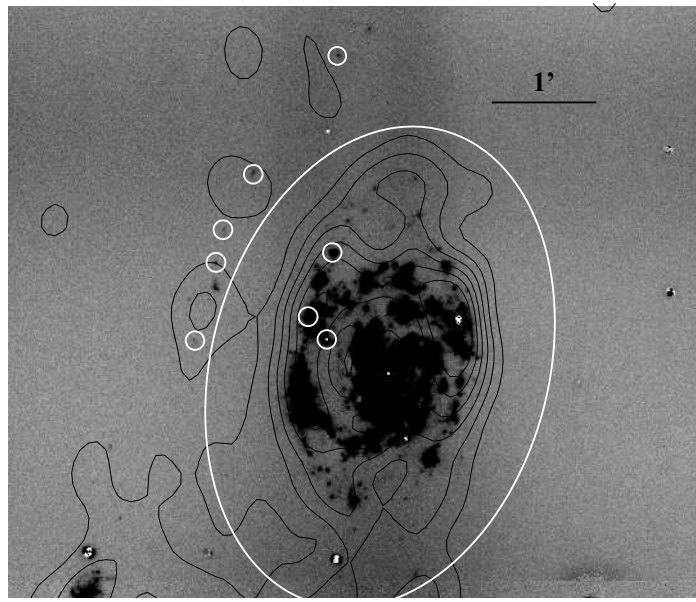

(i) NGC 3893; with $\mathrm{HI}$ contours at 3.5 to $17.0 \mathrm{M}_{\odot} \mathrm{pc}^{-2}$

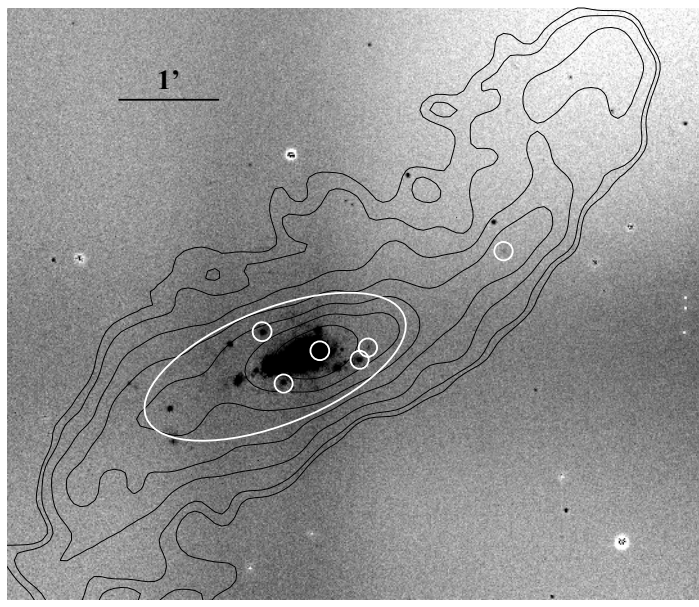

(k) NGC 6239; with HI contours at 2.0 to 25 $\mathrm{M}_{\odot} \mathrm{pc}^{-2}$

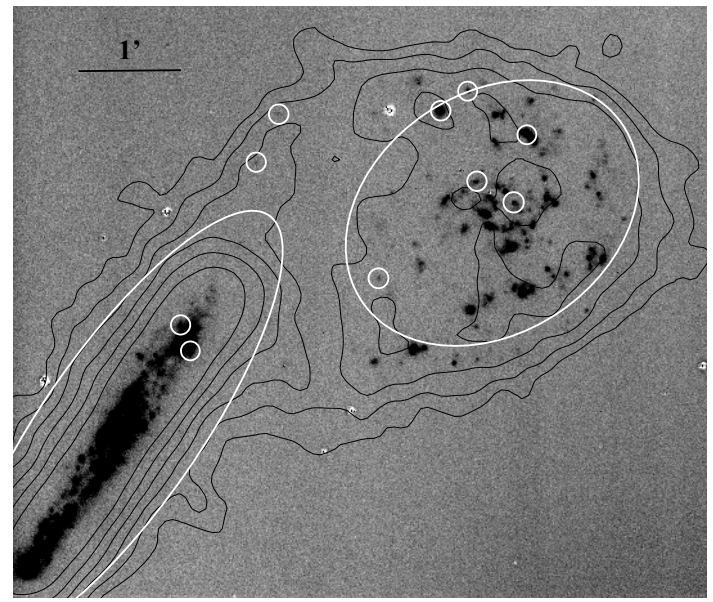

(j) NGC 5774/5; with HI contours at 2.8 to $25 \mathrm{M}_{\odot} \mathrm{pc}^{-2}$

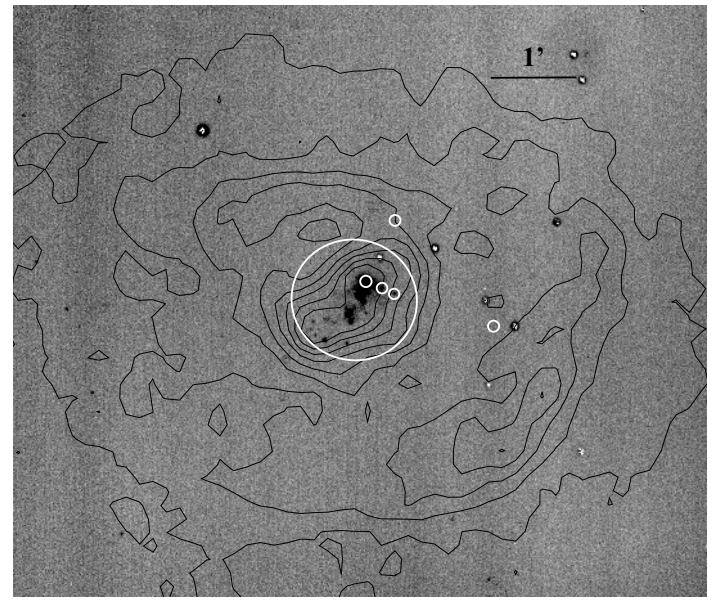

(1) UGC 5288; with HI contours at 0.25 to $6.0 \mathrm{M}_{\odot} \mathrm{pc}^{-2}$

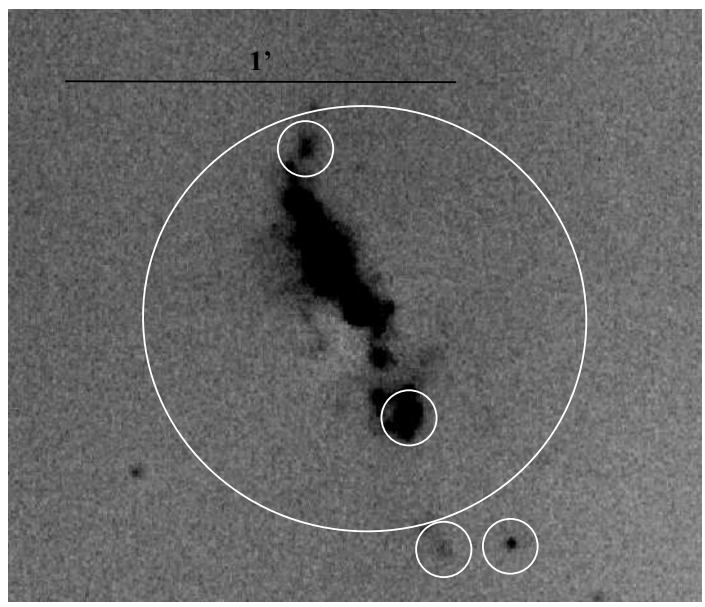

(m) UGC 9562

Figure 1. (Continued)

otherwise "weird-featured" gas-rich galaxies. Of the 13 galaxies (or galaxy pairs) studied here, 10 are mergers or remnants of mergers with extended gaseous features. Both NGC 3359 and UGC 5288 are galaxies with visible SF contained in a much larger, more extended concentration of $\mathrm{H}$ I gas which may or may not be the result of some past interaction despite very regular kinematic structures. NGC 3718 exhibits a large warp in its H I distribution of unknown origin. As we discuss in Section 5, each 
Table 1

Properties of the Galaxies in the H I Rogues Sample

\begin{tabular}{|c|c|c|c|c|c|c|c|c|c|c|c|c|c|}
\hline $\begin{array}{l}\text { Name } \\
(1)\end{array}$ & $\begin{array}{l}\text { R.A. } \\
(2)\end{array}$ & $\begin{array}{c}\text { Decl. } \\
\text { (3) }\end{array}$ & $\begin{array}{c}d \\
(4) \\
\end{array}$ & $\begin{array}{r}R_{25} \\
(5) \\
\end{array}$ & $\begin{array}{c}E(B-V) \\
(6)\end{array}$ & $\begin{array}{l}\text { Abun } \\
(7)\end{array}$ & $\begin{array}{c}{\left[\mathrm{N}_{\text {III }}\right] / \mathrm{H} \alpha} \\
(8)\end{array}$ & $\begin{array}{l}\text { H I Morph } \\
(9)\end{array}$ & $\begin{array}{c}\text { Beam Size } \\
(10)\end{array}$ & $\begin{array}{l}\text { H I Reference } \\
\text { (11) }\end{array}$ & $\begin{array}{r}M_{*} \\
(12) \\
\end{array}$ & $\begin{array}{l}M_{\mathrm{H}_{\mathrm{I}}} \\
(13)\end{array}$ & $\begin{array}{c}\mathrm{SFR}_{\text {tot }} \\
(14)\end{array}$ \\
\hline NGC 2146 & 061838.1 & +782126.3 & 22.45 & 22.15 & 0.096 & $8.68^{1}$ & $0.47^{1}$ & mMR & $77 \times 49$ & Taramopoulos et al. (2001) & 6.02 & 2.10 & 2.2 \\
\hline NGC 2782 & 091405.1 & +400648.9 & 37.30 & 12.93 & 0.016 & $8.80^{1}$ & $0.47^{1}$ & $\mathrm{mMR}$ & $9.4 \times 8.7$ & Smith (1994) & 1.55 & 0.42 & 1.2 \\
\hline NGC 3227 & 102329.0 & +195242.3 & 20.85 & 20.57 & 0.023 & $8.60^{2}$ & $0.30^{2}$ & $\mathrm{E}-\mathrm{Sp}$ & $80 \times 47$ & Mundell (2001) & 5.17 & 0.20 & 0.47 \\
\hline NGC 3239 & 102504.3 & +170911.5 & 8.10 & 6.26 & 0.032 & $8.19^{1}$ & $0.07^{1}$ & $\mathrm{Sp}^{+}-\mathrm{Sp}^{+}$ & $64.3 \times 54.6$ & Iyer et al. (2001) & 0.122 & 0.12 & 0.29 \\
\hline NGC 3310 & 103844.3 & +533007.9 & 18.10 & 9.17 & 0.022 & $8.40^{1}$ & $0.23^{1}$ & mMR & $20 \times 20$ & Iyer et al. (2001) & 0.798 & 0.52 & 3.0 \\
\hline NGC 3359 & 104637.7 & +631325.5 & 17.80 & 13.05 & 0.008 & $8.60^{3}$ & $0.15^{3}$ & Hic & $30 \times 30$ & Boonyasait et al. (2001) & 1.31 & 0.45 & 0.89 \\
\hline NGC 3432 & 105231.2 & +363706.9 & 12.98 & 12.46 & 0.013 & $8.30^{1}$ & $0.12^{1}$ & $\mathrm{mM}$ & $30 \times 30$ & Swaters et al. (2002) & 0.313 & 0.60 & 0.50 \\
\hline NGC 3718 & 113235.0 & +530405.2 & 17.00 & 12.24 & 0.014 & $8.48^{1}$ & $0.22^{1}$ & WARP & $30 \times 30$ & Verheijen \& Sancisi (2001) & 2.94 & 1.00 & 0.25 \\
\hline NGC 3893 & 114838.6 & +48 42 40.8 & 18.13 & 12.81 & 0.021 & $8.60^{1}$ & $0.33^{1}$ & $\mathrm{mM}$ & $30 \times 30$ & Verheijen \& Sancisi (2001) & 2.45 & 0.60 & 1.60 \\
\hline NGC 5774 & 145342.5 & +033456.8 & 26.80 & 12.18 & 0.042 & $8.55^{4}$ & $0.26^{4}$ & $\mathrm{Sp}^{0}-\mathrm{Sp}^{0}$ & $29 \times 23$ & Irwin (1994) & 1.10 & 0.54 & 0.47 \\
\hline NGC 5775 & 145357.2 & +033248.5 & 26.80 & 20.04 & 0.042 & $8.70^{4}$ & $0.36^{4}$ & $\mathrm{Sp}^{0}-\mathrm{Sp}^{0}$ & $29 \times 23$ & Irwin (1994) & 1.36 & 0.91 & 0.70 \\
\hline NGC 6239 & 165005.9 & +424418.9 & 22.30 & 8.92 & 0.018 & $8.40^{4}$ & $0.15^{4}$ & $\mathrm{mM}$ & $18.2 \times 16.7$ & Hogg \& Roberts (2001) & 0.403 & 0.70 & 0.94 \\
\hline UGC 5288 & 095117.2 & +074937.1 & 6.03 & 1.37 & 0.034 & $8.08^{5}$ & $0.07^{5}$ & ENVL & $20.2 \times 16.9$ & van Zee \& Haynes (2001) & 0.00852 & 0.023 & 0.0063 \\
\hline UGC 9562 & 145114.4 & +353232.0 & 23.80 & 3.81 & 0.013 & $8.64^{6}$ & $0.22^{6}$ & $\mathrm{Sp}^{0}-\mathrm{Sp}^{0}$ & $22.5 \times 20.0$ & Cox et al. (2001) & 0.095 & 0.082 & 0.15 \\
\hline
\end{tabular}

Notes. (1) NGC name of the galaxies. (2) R.A. (J2000) in hms. (3) Decl. (J2000) in dms. (4) Redshift-independent distances from NED, in Mpc. (5) Length of the 25th magnitude elliptical isophote $\left(R_{25}\right)$ semimajor axis, in kpc. (6) Color excess $E(B-V)$ from Schlegel et al. (1998). (7) and (8) Oxygen abundances (average or integrated) as $12+\log (\mathrm{O} / \mathrm{H})$ and $[\mathrm{N} \mathrm{II}] / \mathrm{H} \alpha$ ratios from various sources: 1. Moustakas \& Kennicutt (2006): strong-line R23 abundances and [N II]/H $\alpha$ ratios from integrated spectroscopy. 2. Lisenfeld et al. (2008): strong-line R23 abundances and [N II] $/ \mathrm{H} \alpha$ ratios from one $\mathrm{H}$ II region at $0.8 \times R_{25} .3$. Martin \& Roy (1995): average of R23 oxygen abundances and $[\mathrm{N}$ II] $/ \mathrm{H} \alpha$ ratios for disk $\mathrm{H}$ II regions located between 4 and $9.5 \mathrm{kpc}$ in projected distance from the optical center. 4 . Márquez et al. (2002): average of strong-line [N II]/H $\alpha$ abundances and ratios (Pettini \& Pagel 2004) for a number of disk H II regions. 5. van Zee \& Haynes (2006): average strong-line R23 oxygen abundances and [N II]/H $\alpha$ ratios for six H II regions within $R_{25}$. 6. Shi et al. (2005): average strong-line R23 oxygen abundances and [N II]/H $\alpha$ ratios for $\mathrm{H}$ II regions within $R_{25}$. (9) $\mathrm{H}$ I morphology code from the online $\mathrm{H}$ I Rogues catalog-mMR: minor merger remnants; E-Sp: interacting doubles, only one with $\mathrm{H} \mathrm{I}_{\text {; }} \mathrm{Sp}^{+}-\mathrm{Sp}^{+}$: interacting doubles, both with $\mathrm{H}$ I, both with tails; H Ic: detached H I clouds; mM: minor mergers; WARP: galaxies with two-sided warps; $\mathrm{Sp}^{0}-\mathrm{Sp}^{0}$ : interacting doubles, both with $\mathrm{H}$ I, no tails; ENVL: galaxies with extended $\mathrm{H}$ I envelopes. (10) Beam size of $\mathrm{H}$ I observations, in arcseconds. (11) Reference for the H I data. (12) Stellar masses derived from $B$-, $V$-, and sometimes $R$-band photometry and the luminosity/color and stellar mass functions of Bell et al. (2003) in units of $10^{10} \mathcal{M}_{\odot}$. (13) $\mathrm{H}_{\text {I }}$ masses for the galaxy in units of $10^{10} \mathcal{M}_{\odot}$, obtained from the literature (see the text for the original sources) and corrected for the galaxy distances we use in Column 5 of this table. (14) Derived SFRs in $\mathcal{M}_{\odot} \mathrm{yr}^{-1}$ from the total H $\alpha$ luminosity obtained from the MDM $2.4 \mathrm{~m}$ images.

Table 2

Galaxies Imaged at MDM Found to have no Outlying H II Regions

\begin{tabular}{lccc}
\hline \hline Galaxy & $d$ & $R_{25}$ & H I Morph \\
$(1)$ & $(2)$ & $(3)$ & $(4)$ \\
\hline NGC 2768 & 20.06 & 25.63 & EpecH \\
NGC 2777 & 18.61 & 2.27 & $\mathrm{mM}$ \\
NGC 3396 & $28.00 *$ & 15.18 & $\mathrm{Sp}^{+}-\mathrm{Sp}^{+}$ \\
NGC 3471 & 28.64 & 9.0 & $\mathrm{mM}$ \\
NGC 3769 & 15.50 & 13.24 & $\mathrm{mM}$ \\
NGC 3998 & 17.85 & 9.00 & EpecH \\
NGC 4088 & 15.80 & 12.32 & $\mathrm{mM}-3$ \\
NGC 4111 & 16.00 & 16.70 & EpecH \\
NGC 4532 & 15.80 & 8.97 & IGH I \\
NGC 4639 & 22.34 & 10.08 & EXT \\
NGC 4789A & 4.14 & 1.98 & ENVL \\
NGC 5916 & 30.60 & 2.18 & Trip+ \\
UGC 8201 & 4.29 & $\ldots$ & MISC \\
VIRGOHI 21 & $14.00 *$ & IGH I \\
\hline
\end{tabular}

Notes. (1) Common name for the galaxy. (2) Redshift-independent distances from NED, in Mpc. “*” indicates no redshift-independent distances available, Hubble-flow distance. (3) Length of the 25th magnitude elliptical isophote $\left(R_{25}\right)$ semimajor axis, in kpc. (4) H I morphology code from the online $\mathrm{H}_{\mathrm{I}}$ Rogues catalog_EpecH: normal early-type galaxies with peculiar H I; mM-3: minor mergers, three body-encounters; IGH I: intergalactic $\mathrm{H}_{\mathrm{I}}$ with no optical counterpart. To clarify, VIRGOHI 21 is one of these intergalactic clouds, while NGC 4532 is an optically detected galaxy surrounded by such Hi debris; Trip+: interacting triples; MISC: miscellaneous. The caption of Table 1 contains additional descriptions of morphology codes.

individual system is at a different stage in the interaction process and the nature of the interaction ranges from minor to major.

To compare our Galaxies with a sample of "H I-normal" galaxies, we use the Hi-selected sample of the SINGG (Meurer et al. 2006). The SINGG sample was selected from the $\mathrm{H}_{\mathrm{I}}$ Parkes All-Sky Survey (HIPASS; Barnes et al. 2001) purely on the basis of $\mathrm{H}$ I mass and recessional velocity. The primary goal of SINGG is to uniformly survey the SF properties of $\mathrm{HI}$-selected galaxies across the entire $\mathrm{HI}$ mass function sampled by HIPASS $\left(\log \left(M_{\mathrm{H}_{\mathrm{I}}} / M_{\odot}\right) \sim 8.0-10.6\right)$ in a way that is blind to previously known optical properties of the sources.

Hanish et al. (2006) used the SINGG Release 1 data to calculate the luminosity densities in $\mathrm{H} \alpha$ and the $R$ band of the local universe for $\mathrm{HI}$-selected galaxies in the SINGG sample. They then derive the fractional contribution to these luminosity densities as a function of various additional parameters (see their Figure 5 and Table 5). Compared to our minimum $\mathcal{M}_{\mathrm{HI}}$, about $12 \%$ and $22 \%$ of the $R$ and $\mathrm{H} \alpha$ luminosity density, respectively, comes from galaxies with lower $\mathcal{M}_{\mathrm{H}_{\mathrm{I}}}$ values, while about $6 \%$ of both luminosity densities comes from galaxies having $\mathcal{M}_{\mathrm{HI}}$ larger than our maximum value. The median $\mathcal{M}_{\mathrm{HI}}=5.3 \times 10^{9} \mathcal{M}_{\odot}$ is slightly higher than the value $\mathcal{M}_{\mathrm{H}_{\mathrm{I}}} \approx 3.6 \times 10^{9} \mathcal{M}_{\odot}$ corresponding to the midpoint contributing to both these luminosity densities. Hence, the $\mathcal{M}_{\mathrm{H} \text { I }}$ values for our sample correspond well to that of SINGG, but are skewed perhaps to slightly larger values. We can also compare $\mathcal{M}_{\star}$ values, if we convert the SINGG $R$-band luminosities to stellar mass. For this rough calculation, we assume $\mathcal{M}_{\star} / L_{R} \approx 1$ and adopt $M_{R}=4.61 \mathrm{ABmag}$ for the Sun's absolute magnitude. Then we find that at most $3 \%$ of the local $R$ and $\mathrm{H} \alpha$ luminosity densities have $\mathcal{M}_{\star}$ lower than our minimum value, while $30 \%$ of the $R$ luminosity density and $13 \%$ of the $\mathrm{H} \alpha$ luminosity density come from galaxies more massive than our largest $\mathcal{M}_{\star}$. The median $\mathcal{M}_{\star}=1.31 \times 10^{10} \mathcal{M}_{\odot}$ for our sample, while the midpoint for the $R$ and $\mathrm{H} \alpha$ luminosity densities is $\mathcal{M}_{\star}=3.3 \times 10^{10} \mathcal{M}_{\odot}$ and $1.4 \times 10^{10} \mathcal{M}_{\odot}$, respectively. Hence 
our sample covers the $\mathcal{M}_{\star}$ range of the SINGG sample fairly well, but may be deficient in the highest $\mathcal{M}_{\star}$ galaxies.

\section{OPTICAL SPECTROSCOPY}

\subsection{Multi-slit Spectroscopy}

In the spring of 2008, we obtained multi-slit spectra of numerous $\mathrm{H}_{\mathrm{II}}$ regions associated with the $13 \mathrm{H}$ I Rogues using the Gemini-North Multi-Object Spectrograph (GMOS-N) in order to make metallicity measurements of outer-disk and/or stripped gas. We were somewhat limited by the $5^{\prime} \times 5^{\prime}$ field of view of GMOS, which restricted us to partial coverage of the Rogue galaxies and their outskirts. The generation of the GMOS slit mask required that we obtain pre-imaging with GMOS-N in queue mode prior to our spectroscopic observations. We used a $90 \AA$ wide $\mathrm{H} \alpha$ filter for GMOS to image the 13 fields in our sample, and in concert with the $R$-band continuum-subtracted MDM images were then able to pick out and obtain precise instrumental positions for outlying and galactic $\mathrm{H}$ II regions. In only a few cases, we selected potential outlying $\mathrm{H}$ iI regions from the Gemini pre-imaging that were not detected in the MDM images. Four to five alignment star holes per-field and slitlet lengths of at least $10^{\prime \prime}$ allowed us to fit $\sim 25$ science slitlets per mask. In practice, however, we had an average of eight usable science slitlets per mask $($ minimum $=4$; maximum $=17$ ) due to $\mathrm{H}$ II region position/alignment conflicts, very faint $\mathrm{H}$ II regions without multiple emission-line detections (discovered postreduction), several blank "sky slitlets" (see below), constraints on the instrument rotator angle due to available guide stars, and an increased slitlet length for the brightest central $\mathrm{H}$ II regions (for proper night-sky emission-line subtraction).

Our spectral observations were taken in queue mode under the following conditions: a gray lunar phase, an image quality of 1".5 (occurring $85 \%$ of the time), and some cloud cover (better than that occurring 50\% of the time). We used the B600-G5303 600 $1 \mathrm{~mm}^{-1}$ grating with various grating angles optimized for each individual slit mask so that key emission lines would not fall in either of the two CCD chip gaps. Central wavelengths range between 4100 and $4800 \AA$. The above grating configuration results in a spectral coverage of $2760 \AA$ and was chosen so that we could detect emission lines between $\sim 3700$ and $5007 \AA$ for the vast majority of the $\mathrm{H}$ II regions on our masks. Several $\mathrm{H}$ II regions falling close to the image edges did not achieve this range, and, for a few of those $\mathrm{H}$ II regions, we were able to detect the $\mathrm{H} \alpha$ and $[\mathrm{N}$ II] $\lambda 6583$ emission lines. However, we generally did not obtain spectral data above $5500 \AA$ for two reasons: (1) the low-resolution grating (R150, which would have given a larger spectral coverage) is not sensitive enough in the blue to obtain a detection of [O II] $\lambda_{2} 3727$ for our faint objects and (2) an additional grating configuration for red spectral coverage would have doubled our observing time and therefore would have reduced the number of targets we could observe with Gemini. Since we opted for only blue spectral coverage, we used the R23 method for obtaining abundances. We use additional longslit data with red spectral coverage to break the degeneracy of the R23 relation (see Section 4.2 for details). 1".0 wide slitlets and 4 (spatial) $\times 2$ (spectral) binning yield a spectral resolution of $1.8 \AA$ pixel $^{-1}$ and a spatial resolution of $0.28 \operatorname{arcsec~pixel}^{-1}$. Our science exposure times were $3 \times 20$ minutes for each field.

Arc-lamp ( $\mathrm{CuAr}$ ) and spectral flat-field calibrations were done between and after each of the science observations. Baseline Gemini calibrations additionally include the observation of one standard star per grating configuration, HZ-44 in our case, over the execution of the entire science program. The main dangers of using a single standard not taken on the same night as the targets are that seeing variability combined with differential refraction will alter the effective sensitivity function, and that the atmospheric extinction curve will change. Fortunately, in every case, our targets (and the standard star) were observed close to zenith, minimizing the effect of seeing variability with differential refraction. Additionally, the variation in atmospheric extinction (or overall throughput) is minimal on Mauna Kea at optical wavelengths (Krisciunas et al. 1987). Nonetheless, our single relative sensitivity function probably subjects our relative line fluxes to higher errors than if we had used at least one standard star on every night data were taken.

For the reduction of the GMOS multi-slit data, we used the IRAF Gemini/GMOS software package, specifically designed for GMOS multi-slit data reduction. Once the basic reduction is performed (GPREPARE, GSFLAT, GSREDUCE), we apply the wavelength solution from GSWAVELENGTH, using GSTRANSFORM, the fits to which had a typical rms of 0.8-1.0 A. The short slit lengths complicated night-sky line subtraction for several of the bright or more extended $\mathrm{H}$ II regions in our sample. Several blank sky slitlets on every mask allowed us to properly subtract the sky for these bright/extended objects whose emission lines occupied the entire length of the slitlet. We therefore did two passes of sky subtraction for each mask data set, one using GSSKYSUBTRACT, and the other using the manual sky subtraction method. Using GEMCOMBINE and GSEXTRACT, we obtained one-dimensional reduced spectra, which we flux calibrated using GSCALIBRATE based on our sensitivity function derived from $\mathrm{HZ}-44$.

As a test of our manual sky subtraction, we compared the two methods using calibrated one-dimensional spectra of the compact, fainter $\mathrm{H}_{\mathrm{II}}$ regions and found that line fluxes were identical. Typical rms noise in the final, reduced spectra ranges from $\sim 9 \times 10^{-18} \mathrm{erg} \mathrm{s}^{-1} \mathrm{~cm}^{-2}$ at $3730 \AA$, to $\sim 2 \times$ $10^{-18} \mathrm{erg} \mathrm{s}^{-1} \mathrm{~cm}^{-2}$ at $5000 \AA$, to $\sim 7 \times 10^{-19} \mathrm{erg} \mathrm{s}^{-1} \mathrm{~cm}^{-2}$ at $6500 \AA$ (when data were redder due to objects being on image edges). These values do vary somewhat for different slit masks / fields, depend on the aperture size, and are noted only to illustrate that the emission-line Poisson noise is not a major contributor to the error. The rms of the fitted sensitivity function was $6.5 \%$, and we estimate that the addition of flux calibration uncertainty, read noise, sky noise, and flat-fielding errors brings our total errors in line flux measurements up to $\sim 10 \%$.

\subsection{Longslit Spectroscopy}

Over the course of three observing runs, one at the MDM $2.4 \mathrm{~m}$ in the winter of 2007 , one at the Keck $10 \mathrm{~m}$ in the spring of 2010, and one at the Shane $3 \mathrm{~m}$ telescope at Lick Observatory in the spring of 2011, we obtained longslit, lowresolution spectra for outlying $\mathrm{H}$ II regions in our sample of H I Rogues with extended SF. We use these spectra in the subsequent analysis to obtain [N II] $\lambda 6583$ and $\mathrm{H} \alpha$ emission lines, since these lines were generally absent in the GMOS multi-slit data. At MDM, we used the low-resolution Mark III spectrograph with the Echelle CCD, employing the 300/5400 grating and a 0.824 arcsec slit width. The $300 / 5400$ grating centered at $6500 \AA$ gives a spectral coverage of $3120-9879 \AA$, a range containing all of the optical emission lines we could hope to detect, and a resulting dispersion of $3.3 \AA$ pixel ${ }^{-1}$. Blind offsets from nearby stars were required for positioning the often very faint ELdots in the slit. As mentioned in Section 2.2, the run on the MDM $2.4 \mathrm{~m}$ telescope was intended 
as a spectroscopic confirmation run, and we obtained spectra for only a dozen ELdots. Exposure times ranged from 300 $\mathrm{s}$ to $2 \times 600 \mathrm{~s}$, depending on the narrowband flux of the ELdot.

At Keck, we were able to use the low-resolution imaging spectrometer (LRIS) for longslit spectroscopy of three $\mathrm{HI}$ Rogues with ELdots, during an observing run which had several target list "holes" at the very start and end of the nights. The data were taken with the $6001 \mathrm{~mm}^{-1}$ grism (blue side) and $4001 \mathrm{~mm}^{-1}$ grating (red side) which gives a spectral coverage between 3000 and $9300 \AA$. Binning the data $2 \times 2$ resulted in a dispersion of 1.2 and $2.3 \AA$ pixel $^{-1}$ for the blue and red cameras, respectively. ELdots were positioned in the $1^{\prime \prime}$ slit precisely by aligning the offset position angle between it and a bright star with the slit angle. In one case, NGC 6239, we were able to align several other galactic $\mathrm{H}$ II regions with the slit position angle as well. A problem with an amplifier on the red camera results in an unusable lower CCD chip on the red side (each side has two chips). Exposure times were $2 \times 600 \mathrm{~s}$ in the blue and $3 \times$ $400 \mathrm{~s}$ in the red.

At Lick, we used the Kast spectrometer on the Shane $3 \mathrm{~m}$ telescope to observe nine remaining outlying $\mathrm{H}$ II regions without red spectral coverage. We used Grism 1(600/4310) on the blue side, the D55 dichroic, and the 600/7500 grating on the red side, which gave full spectral coverage between 3300 and $8800 \AA$. Exposures were taken through light clouds in most cases, and exposure times ranged from 30 minutes to $1.5 \mathrm{hr}$. The same observing strategy of bright-star position angle alignment used at the Keck telescope was employed here, and our overall success rate of acquiring the faint outlying $\mathrm{H}$ II regions was $100 \%$.

Basic reduction steps, which included bias-subtraction, flatfielding, and flux calibration with a single spectrophotometric standard star (one for each telescope), were performed with the IRAF longslit package for MDM data and with the LowRedux ${ }^{6}$ IDL software package for the Keck and Lick data. We use these longslit data only to obtain $[\mathrm{N}$ II $] / \mathrm{H} \alpha$ ratios in order to break the R23 degeneracy (as described in Section 4.2) for our blue GMOS multi-slit data. Because these two emission lines ([N II] $\lambda 6583$ and $\mathrm{H} \alpha$ at $\lambda=6563 \AA$ ) are so close in wavelength, we did not require an accurate flux (or wavelength) calibration or reddening correction. Table 3 lists the outlying $\mathrm{H}$ II regions for which we were able to observe the $[\mathrm{NII}] / \mathrm{H} \alpha$ ratio using the longslit data. Table 3 also lists the outlying $\mathrm{H}$ II regions so far to the edge of the CCD in the GMOS data for NGC 2146 and NGC 2782 that we were able to obtain $[\mathrm{N} \mathrm{II}] / \mathrm{H} \alpha$ ratios.

\section{ANALYSIS}

\subsection{Emission-line Measurements}

The H II regions in our final sample and marked in Figure 1 were included based on the presence of the relevant emission lines for R23 strong-line abundances. A direct measurement of the H II region's oxygen abundance from the oxygen emission lines present in its spectrum requires a measurement of the temperature-sensitive line ratio [O III] $\lambda \lambda 4959,5007 / \lambda 4363$ (Osterbrock 1989). In these spectra, the critical emission line [O III] $\lambda 4363$ is not detected. Therefore, we use the $R_{23}=([\mathrm{O}$ II $]$ $\lambda 3727+[\mathrm{O}$ III $] \lambda \lambda 4959,5007) / \mathrm{H} \beta$ (Pagel et al. 1979) calibration of McGaugh (1991) to determine the oxygen abundance for

\footnotetext{
6 http://www.ucolick.org/ xavier/LowRedux/index.html
}

Table 3

[N II]/H $\alpha$ for Selected H II Regions from Longslit Spectra Obtained at the MDM, Keck, and Lick Observatories

\begin{tabular}{|c|c|c|c|c|}
\hline $\begin{array}{l}\text { Galaxy } \\
\text { (1) }\end{array}$ & $\begin{array}{c}\text { Region ID } \\
\text { (2) }\end{array}$ & $\begin{array}{c}R / R_{25} \\
\text { (3) }\end{array}$ & $\begin{array}{c}\text { Telescope } \\
(4)\end{array}$ & $\begin{array}{c}{\left[\mathrm{N}_{\mathrm{II}}\right] / \mathrm{H} \alpha} \\
(5)\end{array}$ \\
\hline NGC2146 & 14 & 0.5 & GMOS & $0.31 \pm 0.03$ \\
\hline NGC2146 & 13 & 0.4 & GMOS & $0.31 \pm 0.03$ \\
\hline NGC2146 & 12 & 0.4 & GMOS & $0.27 \pm 0.03$ \\
\hline NGC 2782 & 2 & 2.5 & MDM & $0.24 \pm 0.02$ \\
\hline NGC 2782 & 4 & 1.4 & GMOS & $0.27 \pm 0.02$ \\
\hline NGC 2782 & 6 & 0.6 & GMOS & $0.21 \pm 0.02$ \\
\hline NGC 3239 & 4 & 1.3 & Lick & $0.07 \pm 0.01$ \\
\hline NGC 3310 & 7 & 1.5 & Lick & $0.31 \pm 0.04$ \\
\hline NGC 3310 & 12 & 1.3 & Keck & $0.18 \pm 0.01$ \\
\hline NGC 3359 & 11 & 1.4 & Lick & $0.08 \pm 0.01$ \\
\hline NGC 3359 & 14 & 1.1 & Lick & $0.09 \pm 0.01$ \\
\hline NGC 3432 & 14 & 1.0 & Lick & $0.11 \pm 0.01$ \\
\hline NGC 3718 & 15 & 1.4 & MDM & $0.20 \pm 0.03$ \\
\hline NGC 3718 & 4 & 1.7 & Lick & $0.11 \pm 0.02$ \\
\hline NGC 3893 & 7 & 1.4 & MDM & $0.13 \pm 0.02$ \\
\hline NGC 5774/5 & 4 & 1.5 & MDM & $0.18 \pm 0.02$ \\
\hline NGC 5774/5 & 7 & 1.1 & Lick & $0.18 \pm 0.02$ \\
\hline NGC 5774/5 & 12 & 1.0 & Lick & $0.12 \pm 0.02$ \\
\hline NGC 6239 & 5 & 1.9 & Keck & $0.08 \pm 0.02$ \\
\hline NGC 6239 & $* *$ & 0.6 & Keck & $0.11 \pm 0.02$ \\
\hline NGC 6239 & $* *$ & 0.4 & Keck & $0.22 \pm 0.01$ \\
\hline NGC 6239 & 17 & 0.3 & Keck & $0.12 \pm 0.01$ \\
\hline NGC 6239 & $* *$ & 0.2 & Keck & $0.20 \pm 0.02$ \\
\hline NGC 6239 & $* *$ & 0.1 & Keck & $0.10 \pm 0.01$ \\
\hline UGC 5288 & 1 & 1.4 & Keck & $<0.08$ \\
\hline UGC 5288 & 10 & 0.4 & Keck & $0.06 \pm 0.01$ \\
\hline UGC 9562 & 7 & 1.2 & Keck & $0.08 \pm 0.01$ \\
\hline UGC 9562 & 14 & 0.5 & Keck & $0.08 \pm 0.01$ \\
\hline
\end{tabular}

Notes. Values are also tabulated for those $\mathrm{H}$ II regions with emission lines redward of $\mathrm{H} \alpha$ in the GMOS multi-slit data. (1) Host galaxy name. (2) Region name that corresponds to the GMOS multi-slit ID given in $\mathrm{H}$ II region properties tables, if applicable. (3) Projected distance from the 25th magnitude elliptical isophote. (4) The telescope at which the spectral data were obtained. (5) The ratio of [N II] $\lambda 6583$ to $\mathrm{H} \alpha$ with associated errors. In one case, this value is an upper limit. We use these ratios to break the R23 degeneracy for the Gemini data.

numerous $\mathrm{H}$ II regions in $13 \mathrm{H}$ I Rogue galaxies. The majority of the multi-slit spectra contained $\mathrm{H} \gamma$ as well, which we used to correct for interstellar reddening (see below). The highest signal-to-noise spectra on every mask often contained additional emission lines, including $\mathrm{H} \delta$, $\mathrm{He}_{\mathrm{I}} \lambda 4026$, and $\mathrm{He}$ II $\lambda 4687$, occasionally [Ne III] $\lambda 3869$ and $\lambda 3970$, and rarely the very faint, temperature-sensitive [O III] $\lambda 4363$ auroral emission line which provides a direct oxygen abundance measure. Using the IRAF task splot, and fitting Gaussian profiles we obtain integrated line fluxes. For the inner $\mathrm{H}$ II regions with substantial underlying continuum, we corrected for absorption from underlying stellar populations by subtracting Gaussian fits to the Balmer line absorption.

We apply a correction for interstellar reddening to all line measurements from the observed $\mathrm{H} \gamma$ to $\mathrm{H} \beta$ ratio for case $\mathrm{B}$ recombination where $\mathrm{H} \gamma / \mathrm{H} \beta=0.459$ at an effective temperature of $10,000 \mathrm{~K}$ and electron density of $100 \mathrm{~cm}^{-3}$ (Hummer $\&$ Storey 1987). These lines have been corrected for underlying Balmer absorption. We use a reddening function normalized at $\mathrm{H} \beta$ from the Galactic reddening law of Cardelli et al. (1989) assuming $R_{v}=A_{v} / E(B-V)=3.1$. The mean $E(B-V)$ from the Balmer decrement for these H II regions is 0.252 . We do note additionally that the reddening has very little impact 
Table 4

H II Region Properties for NGC 2146, Sorted by $R / R_{25}$

\begin{tabular}{|c|c|c|c|c|c|c|c|c|c|}
\hline $\begin{array}{l}\text { ID } \\
\text { (1) }\end{array}$ & $\begin{array}{l}\text { R.A. } \\
\text { (2) }\end{array}$ & $\begin{array}{c}\text { Decl. } \\
\text { (3) }\end{array}$ & $\begin{array}{l}R / R_{25} \\
\quad(4)\end{array}$ & $\begin{array}{c}R_{\text {proj }} \\
(5)\end{array}$ & $\begin{array}{c}F_{\mathrm{H} \alpha} \\
(6) \\
\end{array}$ & $\begin{array}{l}m_{R} \\
(7)\end{array}$ & $\begin{array}{c}{\left[\mathrm{O}_{\mathrm{II}}\right]} \\
(8)\end{array}$ & $\begin{array}{c}{[\mathrm{O} \text { III }]} \\
(9)\end{array}$ & $\begin{array}{c}12+\log (\mathrm{O} / \mathrm{H}) \\
(10)\end{array}$ \\
\hline 5 & 061814.9 & +78 1948.4 & 1.5 & 13.12 & $4.13 \pm .49$ & $21.49 \pm .08$ & 226 & 147 & $7.76 / 8.80$ \\
\hline 4 & 061821.8 & +78 1938.2 & 1.4 & 12.94 & $1.11 \pm .13$ & $22.93 \pm .19$ & 243 & 466 & $8.04 / 8.56$ \\
\hline 8 & 061809.1 & +782102.4 & 1.1 & 9.91 & $2.98 \pm .35$ & $22.14 \pm .11$ & 328 & 137 & $7.97 / 8.70$ \\
\hline 9 & 061804.4 & +782123.3 & 1.1 & 11.13 & $1.66 \pm .19$ & $20.64 \pm .07$ & 267 & 64 & $7.84 / 8.82$ \\
\hline 14 & 061900.5 & +782044.1 & 0.5 & 8.68 & $3.57 \pm .42$ & $21.22 \pm .08$ & $* * *$ & $* * *$ & $8.61 *$ \\
\hline 15 & 061852.5 & +782037.8 & 0.3 & 7.09 & $4.44 \pm .53$ & $22.94 \pm .19$ & 161 & 79 & 8.92 \\
\hline
\end{tabular}

Notes. Description of columns applies to Tables 4- 16. (1) The slit mask ID for the H II region. (2) Right ascension (J2000) in hours, minutes, and seconds. (3) Declination (J2000) in degrees, minutes, and seconds. (4) Projected distance from the 25th magnitude elliptical isophote. (5) Projected distance in kpc from the center of the galaxy. (6) $\mathrm{H} \alpha$ flux and associated error from MDM $2.4 \mathrm{~m}$ images in units of $10^{-15} \mathrm{erg} \mathrm{s}^{-1} \mathrm{~cm}^{-2}$. (7) $R$-band apparent magnitude and associated error from MDM $2.4 \mathrm{~m}$ images in AB magnitudes. We use the $5 \sigma$ limiting magnitude for a point source in each image when an $\mathrm{H}$ II region is undetected in the $R$ band. (8) and (9) The reddening-corrected [O II] $\lambda \lambda 3727$ and [O III] $\lambda 4959$ and $\lambda 5007$ summed emission-line strengths, relative to $\mathrm{H} \beta$ of 100 . Reddening correction was done for case $\mathrm{B}$ recombination with $T=10,000 \mathrm{~K}$ and $n_{e}=100 \mathrm{~cm}^{-3}$, where $\mathrm{H} \alpha / \mathrm{H} \beta=2.86$ and $\mathrm{H} \gamma / \mathrm{H} \beta=0.459$. (10) The strong-line oxygen abundance, $12+\log (\mathrm{O} / \mathrm{H})$, from the M91 calibration of the R23 relation described in Section 4. In cases where there is one value, we have used the [N II]/H $\alpha$ to break the R23 degeneracy. In cases where there are two values, the $[\mathrm{N} \mathrm{II}] / \mathrm{H} \alpha$ either falls in the ambiguous range $(0.05-0.08)$ or was not available. The first value is the lower-branch R23 oxygen abundance, the second is the upper-branch R23 oxygen abundance. The error associated with strong-line abundances is notoriously large, ranging from 0.15 to 0.2 dex, as discussed in Section 4. In a few cases (for just NGC 2146 and NGC 2782), values for $12+\log (\mathrm{O} / \mathrm{H})$ marked with an asterisk indicate that the GMOS multi-slit spectra were red enough to obtain $\mathrm{H} \alpha$ and $[\mathrm{N} \mathrm{II}] \lambda 6583$, but not the blue lines. In these few cases, we used the $[\mathrm{N}$ II] $/ \mathrm{H} \alpha$ method from PP04 to determine the oxygen abundance. These values cannot be compared directly with R23 values because of systematic offsets of the calibration methods.

Table 5

H II Region Properties: NGC 2782

\begin{tabular}{|c|c|c|c|c|c|c|c|c|c|}
\hline $\begin{array}{l}\text { ID } \\
(1) \\
\end{array}$ & $\begin{array}{l}\text { R.A. } \\
(2)\end{array}$ & $\begin{array}{l}\text { Decl. } \\
\text { (3) }\end{array}$ & $\begin{array}{c}R / R_{25} \\
(4) \\
\end{array}$ & $\begin{array}{c}R_{\text {proj }} \\
(5)\end{array}$ & $\begin{array}{c}F_{\mathrm{H} \alpha} \\
(6) \\
\end{array}$ & $\begin{array}{l}m_{R} \\
(7) \\
\end{array}$ & $\begin{array}{c}{[\mathrm{O} \text { II }]} \\
(8)\end{array}$ & $\begin{array}{c}{[\mathrm{O} \text { III] }} \\
(9)\end{array}$ & $\begin{array}{c}12+\log (\mathrm{O} / \mathrm{H}) \\
(10) \\
\end{array}$ \\
\hline 1 & 091350.9 & +400816.4 & 2.6 & 33.51 & $0.44 \pm .05$ & $23.07 \pm .30$ & 391 & 80 & $8.66^{*}$ \\
\hline 2 & 091351.2 & +400806.8 & 2.5 & 32.14 & $1.71 \pm .20$ & $22.46 \pm .19$ & 338 & 84 & $8.72 *$ \\
\hline 4 & 091358.8 & +400608.8 & 1.2 & 15.00 & $0.28 \pm .03$ & $>23.72 \pm .36$ & $* * *$ & $* * *$ & 8.55 \\
\hline 6 & 091401.4 & +400636.3 & 0.6 & 7.99 & $1.40 \pm .16$ & $21.96 \pm .13$ & $* * *$ & $* * *$ & 8.47 \\
\hline
\end{tabular}

Note. Same as Table 4, for NGC 2782.

Table 6

H II Region Properties: NGC 3227

\begin{tabular}{|c|c|c|c|c|c|c|c|c|c|}
\hline $\begin{array}{l}\text { ID } \\
(1)\end{array}$ & $\begin{array}{l}\text { R.A. } \\
(2)\end{array}$ & $\begin{array}{c}\text { Decl. } \\
\text { (3) }\end{array}$ & $\begin{array}{c}R / R_{25} \\
(4)\end{array}$ & $\begin{array}{c}R_{\text {proj }} \\
(5)\end{array}$ & $\begin{array}{l}F_{\mathrm{H} \alpha} \\
(6)\end{array}$ & $\begin{array}{l}m_{R} \\
(7) \\
\end{array}$ & $\begin{array}{c}{[\mathrm{O} \text { II }]} \\
(8)\end{array}$ & $\begin{array}{c}{[\mathrm{O} \text { III }]} \\
(9)\end{array}$ & $\begin{array}{c}12+\log (\mathrm{O} / \mathrm{H}) \\
(10)\end{array}$ \\
\hline 5 & 102335.1 & +195307.9 & 1.1 & 9.08 & $0.57 \pm .06$ & $22.75 \pm .16$ & 402 & 115 & 8.63 \\
\hline 24 & 102325.2 & +195144.4 & 1.0 & 7.95 & $7.21 \pm .86$ & $20.61 \pm .07$ & 330 & 141 & 8.69 \\
\hline 10 & 102326.4 & +195414.5 & 0.5 & 10.04 & $0.87 \pm .10$ & $20.93 \pm .07$ & 461 & 85 & 8.58 \\
\hline 12 & 102326.1 & +195401.9 & 0.5 & 9.03 & $0.93 \pm .11$ & $19.45 \pm .07$ & 446 & 124 & 8.57 \\
\hline 22 & 102332.9 & +195154.3 & 0.4 & 7.39 & $1.42 \pm .17$ & $21.15 \pm .07$ & 234 & 50 & 8.87 \\
\hline
\end{tabular}

Note. Same as Table 4, for NGC 3227.

on the calculated strong-line abundances, which have very large errors. Tables 4-16 present the reddening-corrected strong-line measurements for the $\mathrm{H}$ II regions in all 13 Rogue galaxies. All listed line fluxes are relative to $\mathrm{H} \beta=100$.

\subsection{Breaking the Degeneracy of the R23 Relation}

We use the McGaugh (1991, henceforth M91) calibration of the $\mathrm{R} 23$ relation to obtain the oxygen abundances of all the $\mathrm{H}$ II regions in our sample of 13 Rogue systems. The $\mathrm{R} 23$ relation exhibits a well-known degeneracy, with a turnover in the relation at $Z \sim 0.3 Z_{\odot}(12+\log (\mathrm{O} / \mathrm{H}) \sim 8.35)$. The most robust way to place an $\mathrm{H}$ II region on the upper or lower branch of the R23 relation is to use the $\left[\mathrm{N}\right.$ II] $\lambda 6583$ to $\left[\mathrm{O}_{\text {II }}\right] \lambda \lambda 3727$ ratio. When $\log [\mathrm{N}$ II $] /\left[\mathrm{O}_{\mathrm{II}}\right]<-1.0, \mathrm{H}$ II regions lie on the lower metallicity branch of the R23 relation (Kewley \& Ellison 2008). Since our multi-slit data are bluer than $5500 \AA$ in most cases, we cannot use this method. Instead, we use the $[\mathrm{N}$ II $] / \mathrm{H} \alpha$ ratio as described in Kewley \& Ellison (2008) from our longslit spectra and from the literature. The primary benefit of this line ratio is that it does not require an accurate (or absolute) flux calibration or reddening correction due to the proximity in wavelength of $[\mathrm{N}$ II] $\lambda 6583$ and $\mathrm{H} \alpha$. This benefit is important for our data redward of $5600 \AA$, since we are using spectra from four different telescopes taken under varying conditions. 
Table 7

H II Region Properties: NGC 3239

\begin{tabular}{|c|c|c|c|c|c|c|c|c|c|}
\hline $\begin{array}{l}\text { ID } \\
(1)\end{array}$ & $\begin{array}{l}\text { R.A. } \\
(2)\end{array}$ & $\begin{array}{c}\text { Decl. } \\
\text { (3) }\end{array}$ & $\begin{array}{c}R / R_{25} \\
\quad(4)\end{array}$ & $\begin{array}{c}R_{\text {proj }} \\
(5)\end{array}$ & $\begin{array}{c}F_{\mathrm{H} \alpha} \\
(6)\end{array}$ & $\begin{array}{l}m_{R} \\
(7)\end{array}$ & $\begin{array}{c}{[\mathrm{O} \text { II }]} \\
(8)\end{array}$ & $\begin{array}{c}{[\mathrm{O} \text { III] }} \\
(9)\end{array}$ & $\begin{array}{c}12+\log (\mathrm{O} / \mathrm{H}) \\
(10)\end{array}$ \\
\hline 4 & 102509.4 & +170719.9 & 1.3 & 5.23 & $1.10 \pm .13$ & $21.69 \pm .08$ & 360 & 172 & $8.03 / 8.64$ \\
\hline 5 & 102456.2 & +171013.3 & 1.1 & 5.17 & $0.27 \pm .03$ & $23.54 \pm .21$ & 363 & 47 & $8.08 / 8.72$ \\
\hline 13 & 102507.3 & +170741.1 & 1.0 & 3.92 & $0.89 \pm .10$ & $22.48 \pm .10$ & 280 & 400 & $8.06 / 8.56$ \\
\hline 24 & 102503.0 & +170752.6 & 0.8 & 3.19 & $2.65 \pm .31$ & $21.77 \pm .08$ & 262 & 324 & $7.97 / 8.63$ \\
\hline 14 & 102503.3 & +170805.8 & 0.7 & 2.65 & $0.35 \pm .04$ & $23.15 \pm .16$ & 404 & 166 & $8.10 / 8.59$ \\
\hline 22 & 102502.7 & +171000.8 & 0.6 & 2.13 & $2.53 \pm .30$ & $21.07 \pm .07$ & 204 & 231 & $7.78 / 8.75$ \\
\hline 17 & 102458.8 & +170929.3 & 0.6 & 3.15 & $2.63 \pm .31$ & $20.94 \pm .07$ & 348 & 331 & $8.12 / 8.54$ \\
\hline 21 & 102506.8 & +170957.5 & 0.5 & 2.29 & $3.77 \pm .45$ & $20.84 \pm .07$ & 455 & 189 & 8.20 \\
\hline 19 & 102500.2 & +170938.7 & 0.5 & 2.53 & $3.01 \pm .36$ & $20.75 \pm .07$ & 446 & 215 & 8.21 \\
\hline
\end{tabular}

Note. Same as Table 4, for NGC 3239.

Table 8

H II Region Properties: NGC 3310

\begin{tabular}{|c|c|c|c|c|c|c|c|c|c|}
\hline $\begin{array}{l}\text { ID } \\
(1) \\
\end{array}$ & $\begin{array}{c}\text { R.A. } \\
\text { (2) }\end{array}$ & $\begin{array}{l}\text { Decl. } \\
\text { (3) }\end{array}$ & $\begin{array}{c}R / R_{25} \\
(4)\end{array}$ & $\begin{array}{l}R_{\text {proj }} \\
(5)\end{array}$ & $\begin{array}{c}F_{\mathrm{H} \alpha} \\
(6) \\
\end{array}$ & $\begin{array}{l}m_{R} \\
(7) \\
\end{array}$ & $\begin{array}{c}{[\mathrm{O} \text { II }]} \\
(8)\end{array}$ & $\begin{array}{c}{[\mathrm{O} \text { III] }} \\
(9)\end{array}$ & $\begin{array}{c}12+\log (\mathrm{O} / \mathrm{H}) \\
(10)\end{array}$ \\
\hline 11 & 103827.2 & +533158.6 & 1.8 & 16.54 & $0.06 \pm .00$ & $>23.79 \pm .35$ & 319 & 90 & 8.74 \\
\hline 7 & 103831.0 & +533146.5 & 1.5 & 13.54 & $0.33 \pm .03$ & $23.01 \pm .19$ & 302 & 88 & 8.76 \\
\hline 12 & 103832.4 & +533125.7 & 1.3 & 11.54 & $1.40 \pm .16$ & $21.54 \pm .08$ & 421 & 184 & 8.56 \\
\hline 14 & 103833.8 & +533116.7 & 1.1 & 10.19 & $1.43 \pm .17$ & $22.39 \pm .12$ & 411 & 220 & 8.55 \\
\hline 15 & 103834.6 & +533112.3 & 1.0 & 9.49 & $2.40 \pm .28$ & $21.74 \pm .09$ & 341 & 111 & 8.70 \\
\hline 24 & 103841.3 & +533101.7 & 0.6 & 5.27 & $6.52 \pm .78$ & $21.06 \pm .07$ & 127 & 664 & 8.57 \\
\hline 26 & 103844.9 & +533057.2 & 0.5 & 4.34 & $5.85 \pm .70$ & $21.15 \pm .07$ & 288 & 497 & 8.49 \\
\hline 25 & 103843.1 & +533043.8 & 0.4 & 3.27 & $8.34 \pm .00$ & $20.75 \pm .07$ & 140 & 540 & 8.62 \\
\hline
\end{tabular}

Note. Same as Table 4, for NGC 3310.

Table 9

H II Region Properties: NGC 3359

\begin{tabular}{|c|c|c|c|c|c|c|c|c|c|}
\hline $\begin{array}{l}\text { ID } \\
(1) \\
\end{array}$ & $\begin{array}{l}\text { R.A. } \\
(2)\end{array}$ & $\begin{array}{l}\text { Decl. } \\
\text { (3) }\end{array}$ & $\begin{array}{c}R / R_{25} \\
(4)\end{array}$ & $\begin{array}{c}R_{\text {proj }} \\
(5)\end{array}$ & $\begin{array}{c}F_{\mathrm{H} \alpha} \\
(6)\end{array}$ & $\begin{array}{r}m_{R} \\
(7) \\
\end{array}$ & $\begin{array}{c}{[\mathrm{O} \text { II }]} \\
(8)\end{array}$ & $\begin{array}{c}{[\mathrm{O} \text { III] }} \\
(9)\end{array}$ & $\begin{array}{c}12+\log (\mathrm{O} / \mathrm{H}) \\
(10) \\
\end{array}$ \\
\hline 6 & 104610.6 & +631426.8 & 1.5 & 16.65 & $0.26 \pm .02$ & $>23.16 \pm .36$ & 269 & 209 & 8.71 \\
\hline 12 & 104630.9 & +631649.3 & 1.4 & 18.05 & $0.55 \pm .06$ & $22.32 \pm .19$ & 434 & 164 & 8.56 \\
\hline 11 & 104626.6 & +631628.4 & 1.4 & 17.08 & $0.95 \pm .11$ & $22.43 \pm .21$ & 206 & 320 & 8.69 \\
\hline 7 & 104620.1 & +631524.0 & 1.2 & 14.48 & $0.45 \pm .05$ & $>23.16 \pm .36$ & 291 & 51 & 8.80 \\
\hline 8 & 104624.0 & +631548.2 & 1.2 & 14.70 & $0.41 \pm .04$ & $>23.16 \pm .36$ & 230 & 227 & 8.73 \\
\hline 13 & 104637.9 & +631620.2 & 1.2 & 15.09 & $1.85 \pm .22$ & $21.54 \pm .11$ & 454 & 182 & 8.53 \\
\hline 14 & 104639.6 & +631610.3 & 1.1 & 14.28 & $7.59 \pm .91$ & $20.75 \pm .08$ & 158 & 555 & 8.59 \\
\hline 17 & 104626.7 & +631505.4 & 0.9 & 10.74 & $3.99 \pm .47$ & $21.18 \pm .09$ & 425 & 213 & 8.54 \\
\hline 16 & 104637.6 & +631532.4 & 0.8 & 10.96 & $6.95 \pm .83$ & $20.94 \pm .08$ & 221 & 525 & 8.54 \\
\hline 19 & 104651.1 & +631413.9 & 0.7 & 8.86 & $0.78 \pm 0.08$ & $>23.16 \pm .36$ & 210 & 244 & 8.74 \\
\hline 28 & 104628.6 & +631206.9 & 0.7 & 8.60 & $12.08 \pm .44$ & $19.74 \pm .07$ & 576 & 299 & 8.40 \\
\hline 20 & 104644.8 & +631438.1 & 0.6 & 7.51 & $13.90 \pm .66$ & $20.19 \pm .07$ & 247 & 867 & 8.37 \\
\hline 27 & 104631.1 & +631232.7 & 0.5 & 5.94 & $1.92 \pm .22$ & $>23.16 \pm .36$ & 276 & 145 & 8.74 \\
\hline 23 & 104628.6 & +631338.1 & 0.5 & 5.40 & $13.39 \pm .60$ & $20.27 \pm .07$ & 253 & 277 & 8.67 \\
\hline 24 & 104630.7 & +631322.2 & 0.4 & 4.06 & $9.76 \pm .17$ & $20.53 \pm .07$ & 207 & 323 & 8.69 \\
\hline 25 & 104642.8 & +631316.7 & 0.3 & 3.07 & $4.81 \pm .57$ & $20.78 \pm .08$ & 423 & 111 & 8.61 \\
\hline
\end{tabular}

Note. Same as Table 4, for NGC 3359.

Kewley \& Ellison (2008) discuss how to use the $[\mathrm{N}$ II $] \lambda 6583 / \mathrm{H} \alpha$ ratio ( $\mathrm{N} 2$ index) to break the degeneracy in the $\mathrm{R} 23$ relation. If $\log \mathrm{N} 2<-1.3([\mathrm{~N}$ II $] / \mathrm{H} \alpha<0.05)$, there is a high degree of certainty $(\sim 86 \%)$ that the oxygen abundance is on the lower branch of the R23 relation. Whereas if $\log \mathrm{N} 2>$ $-1.1([\mathrm{~N} \mathrm{II}] / \mathrm{H} \alpha>0.08)$, the oxygen abundance is on the up- per branch. Between -1.1 and -1.3 (0.05 and 0.08), $\log (\mathrm{N} 2)$ $(([\mathrm{N} \mathrm{II}] / \mathrm{H} \alpha)$ does not accurately discriminate between the upper and lower branches of $\mathrm{R} 23$ because the oxygen abundance is very close to the turnover at $12+\log (\mathrm{O} / \mathrm{H})=8.3$. This method of breaking the R23 degeneracy works because the N2 index is sensitive to and monotonic with the oxygen abundance to 
Table 10

H II Region Properties: NGC 3432

\begin{tabular}{|c|c|c|c|c|c|c|c|c|c|}
\hline $\begin{array}{l}\text { ID } \\
(1) \\
\end{array}$ & $\begin{array}{l}\text { R.A. } \\
(2)\end{array}$ & $\begin{array}{l}\text { Decl. } \\
\text { (3) }\end{array}$ & $\begin{array}{c}R / R_{25} \\
\quad(4)\end{array}$ & $\begin{array}{c}R_{\text {proj }} \\
(5)\end{array}$ & $\begin{array}{c}F_{\mathrm{H} \alpha} \\
(6)\end{array}$ & $\begin{array}{l}m_{R} \\
(7) \\
\end{array}$ & $\begin{array}{c}{[\mathrm{O} \text { II }]} \\
(8)\end{array}$ & $\begin{array}{c}{[\mathrm{O} \text { III }]} \\
(9)\end{array}$ & $\begin{array}{c}12+\log (\mathrm{O} / \mathrm{H}) \\
(10) \\
\end{array}$ \\
\hline 7 & 105247.2 & +363938.9 & 1.4 & 15.46 & $0.56 \pm .06$ & $23.77 \pm .25$ & 277 & 433 & 8.54 \\
\hline 5 & 105243.4 & +364034.7 & 1.3 & 16.01 & $0.35 \pm .04$ & $23.47 \pm .20$ & 314 & 383 & 8.54 \\
\hline 12 & 105242.1 & +363951.3 & 1.1 & 13.26 & $0.11 \pm .01$ & $>24.20 \pm .36$ & 269 & 514 & 8.50 \\
\hline 14 & 105240.5 & +363952.2 & 1.0 & 12.56 & $0.78 \pm .09$ & $22.36 \pm .10$ & 111 & 297 & 8.80 \\
\hline 17 & 105238.1 & +363846.9 & 0.7 & 8.21 & $0.36 \pm .04$ & $23.16 \pm .16$ & 288 & 695 & 8.30 \\
\hline
\end{tabular}

Note. Same as Table 4, for NGC 3432.

Table 11

H II Region Properties: NGC 3718

\begin{tabular}{|c|c|c|c|c|c|c|c|c|c|}
\hline $\begin{array}{l}\text { ID } \\
\text { (1) } \\
\end{array}$ & $\begin{array}{l}\text { R.A. } \\
\text { (2) }\end{array}$ & $\begin{array}{l}\text { Decl. } \\
\text { (3) }\end{array}$ & $\begin{array}{c}R / R_{25} \\
\quad(4)\end{array}$ & $\begin{array}{c}R_{\text {proj }} \\
(5)\end{array}$ & $\begin{array}{c}F_{\mathrm{H} \alpha} \\
(6)\end{array}$ & $\begin{array}{c}m_{R} \\
(7) \\
\end{array}$ & $\begin{array}{c}{\left[\mathrm{O}_{\mathrm{II}}\right]} \\
(8)\end{array}$ & $\begin{array}{c}{[\mathrm{O} \text { III }]} \\
(9)\end{array}$ & $\begin{array}{c}12+\log (\mathrm{O} / \mathrm{H}) \\
(10)\end{array}$ \\
\hline 4 & 113248.3 & +530655.5 & 1.7 & 17.16 & $0.17 \pm .02$ & $>23.60 \pm .36$ & 393 & 239 & 8.55 \\
\hline 6 & 113248.3 & +530556.8 & 1.4 & 13.46 & $0.31 \pm .03$ & $>23.60 \pm .36$ & 327 & 77 & 8.74 \\
\hline 15 & 113251.5 & +530206.3 & 1.4 & 15.65 & $2.50 \pm .30$ & $21.58 \pm .11$ & 394 & 162 & 8.60 \\
\hline 12 & 113245.7 & +530233.7 & 1.0 & 10.96 & $1.16 \pm .13$ & $22.72 \pm .25$ & 378 & 303 & 8.53 \\
\hline 11 & 113247.2 & +530322.4 & 1.0 & 9.72 & $0.55 \pm .06$ & $>23.60 \pm .36$ & 506 & 114 & 8.51 \\
\hline 8 & 113245.2 & +530407.1 & 0.8 & 7.58 & $0.28 \pm .03$ & $23.01 \pm .32$ & 529 & 267 & 8.40 \\
\hline 7 & 113238.9 & +530543.0 & 0.8 & 8.56 & $0.29 \pm .03$ & $>23.60 \pm .36$ & 322 & 71 & 8.75 \\
\hline 19 & 113241.9 & +530254.6 & 0.7 & 7.73 & $1.81 \pm .21$ & $21.54 \pm .11$ & 266 & 74 & 8.81 \\
\hline 20 & 113240.0 & +530312.0 & 0.5 & 5.71 & $0.96 \pm .11$ & $>23.60 \pm .36$ & 291 & 145 & 8.73 \\
\hline
\end{tabular}

Note. Same as Table 4, for NGC 3718.

Table 12

H II Region Properties: NGC 3893

\begin{tabular}{|c|c|c|c|c|c|c|c|c|c|}
\hline $\begin{array}{l}\text { ID } \\
(1) \\
\end{array}$ & $\begin{array}{l}\text { R.A. } \\
(2)\end{array}$ & $\begin{array}{c}\text { Decl. } \\
\text { (3) }\end{array}$ & $\begin{array}{c}R / R_{25} \\
(4)\end{array}$ & $\begin{array}{c}R_{\text {proj }} \\
(5)\end{array}$ & $\begin{array}{c}F_{\mathrm{H} \alpha} \\
(6) \\
\end{array}$ & $\begin{array}{l}m_{R} \\
(7) \\
\end{array}$ & $\begin{array}{c}{[\mathrm{O} \text { II }]} \\
(8)\end{array}$ & $\begin{array}{c}{[\mathrm{O} \text { III }]} \\
(9) \\
\end{array}$ & $\begin{array}{c}12+\log (\mathrm{O} / \mathrm{H}) \\
(10) \\
\end{array}$ \\
\hline 7 & 114841.2 & +484548.0 & 1.4 & 16.61 & $0.93 \pm .11$ & $22.73 \pm .16$ & 135 & 521 & 8.64 \\
\hline 10 & 114846.3 & +484438.2 & 1.2 & 12.30 & $1.04 \pm .12$ & $22.65 \pm .15$ & 209 & 110 & 8.85 \\
\hline 15 & 114848.5 & +484344.7 & 1.1 & 10.31 & $1.13 \pm .13$ & $22.72 \pm .15$ & 301 & 77 & 8.77 \\
\hline 19 & 114849.8 & +484258.5 & 1.1 & 9.83 & $0.51 \pm .06$ & $23.68 \pm .32$ & 361 & 168 & 8.64 \\
\hline 21 & 114841.5 & +484350.6 & 0.6 & 6.62 & $17.42 \pm .09$ & $19.79 \pm .07$ & 250 & 169 & 8.75 \\
\hline
\end{tabular}

Note. Same as Table 4, for NGC 3893.

Table 13

H II Region Properties: NGC 5774/5

\begin{tabular}{|c|c|c|c|c|c|c|c|c|c|}
\hline $\begin{array}{l}\text { ID } \\
(1)\end{array}$ & $\begin{array}{l}\text { R.A. } \\
(2)\end{array}$ & $\begin{array}{c}\text { Decl. } \\
(3)\end{array}$ & $\begin{array}{c}R / R_{25} \\
\quad(4)\end{array}$ & $\begin{array}{c}R_{\text {proj }} \\
(5)\end{array}$ & $\begin{array}{c}F_{\mathrm{H} \alpha} \\
(6)\end{array}$ & $\begin{array}{l}m_{R} \\
(7) \\
\end{array}$ & $\begin{array}{c}{\left[\mathrm{O}_{\mathrm{II}}\right]} \\
(8)\end{array}$ & $\begin{array}{c}{[\mathrm{O} \text { III] }} \\
(9)\end{array}$ & $\begin{array}{c}12+\log (\mathrm{O} / \mathrm{H}) \\
(10)\end{array}$ \\
\hline \multicolumn{10}{|c|}{ NGC 5774} \\
\hline 7 & 145348.6 & +033414.1 & 1.1 & 13.11 & $0.04 \pm .00$ & $20.20 \pm .07$ & 320 & 300 & 8.59 \\
\hline 12 & 145344.6 & +033558.0 & 1.0 & 8.94 & $5.74 \pm .68$ & $19.85 \pm .07$ & 189 & 616 & 8.53 \\
\hline 23 & 145341.1 & +033543.7 & 0.6 & 6.65 & $10.10 \pm .21$ & $20.06 \pm .07$ & 343 & 276 & 8.58 \\
\hline 10 & 145343.1 & +033516.0 & 0.3 & 2.78 & $1.91 \pm .22$ & $21.92 \pm .13$ & 373 & 293 & 8.54 \\
\hline 4 & 145351.0 & +033555.9 & 1.5 & 27.20 & $0.40 \pm .04$ & $>23.19 \pm .34$ & 439 & 230 & 8.51 \\
\hline 1 & 145351.9 & +033527.0 & 1.3 & 23.05 & $0.54 \pm .06$ & $21.67 \pm .11$ & 396 & 263 & 8.54 \\
\hline 15 & 145354.8 & +033350.7 & 0.5 & 9.29 & $4.22 \pm .50$ & $22.42 \pm .19$ & 320 & 154 & 8.69 \\
\hline 17 & 145354.5 & +033334.4 & 0.4 & 7.98 & $4.80 \pm .57$ & $22.26 \pm .17$ & 270 & 87 & 8.80 \\
\hline
\end{tabular}

Note. Same as Table 4, for NGC 5774 and NGC 5775.

within 0.3 dex accuracy at a $95 \%$ confidence level up to $12+\log (\mathrm{O} / \mathrm{H})=8.8$ (Pettini \& Pagel 2004, henceforth PP04).
The primary drawback of using this method, then, is the large uncertainty in the calibration $(\sim 0.4 \mathrm{dex})$, and the limited range 
Table 14

H II Region Properties: NGC 6239

\begin{tabular}{|c|c|c|c|c|c|c|c|c|c|}
\hline $\begin{array}{l}\text { ID } \\
(1)\end{array}$ & $\begin{array}{l}\text { R.A. } \\
(2)\end{array}$ & $\begin{array}{l}\text { Decl. } \\
(3)\end{array}$ & $\begin{array}{l}R / R_{25} \\
\quad(4)\end{array}$ & $\begin{array}{l}R_{\text {proj }} \\
(5)\end{array}$ & $\begin{array}{c}F_{\mathrm{H} \alpha} \\
(6)\end{array}$ & $\begin{array}{l}m_{R} \\
(7) \\
\end{array}$ & $\begin{array}{c}{[\mathrm{O} \text { II }]} \\
(8)\end{array}$ & $\begin{array}{c}{[\mathrm{O} \text { III }]} \\
(9)\end{array}$ & $\begin{array}{c}12+\log (\mathrm{O} / \mathrm{H}) \\
(10)\end{array}$ \\
\hline 5 & 164953.6 & +424528.2 & 1.9 & 16.42 & $0.46 \pm .05$ & $>23.15 \pm .35$ & 480 & 269 & 8.42 \\
\hline 8 & 165000.9 & +424430.6 & 0.7 & 6.07 & $0.53 \pm .06$ & $>23.15 \pm .35$ & 256 & 150 & 8.76 \\
\hline 9 & 165001.3 & +424423.3 & 0.7 & 5.42 & $2.05 \pm .24$ & $21.80 \pm .13$ & 382 & 313 & 8.52 \\
\hline 11 & 165006.6 & +424440.1 & 0.7 & 2.45 & $3.29 \pm .39$ & $21.01 \pm .08$ & 261 & 170 & 8.74 \\
\hline 17 & 165003.5 & +424428.7 & 0.3 & 3.04 & $73.58 \pm .82$ & $18.36 \pm .07$ & 122 & 698 & 8.55 \\
\hline 16 & 165005.5 & +424410.0 & 0.3 & 1.06 & $2.21 \pm .26$ & $21.62 \pm .11$ & 275 & 321 & 8.62 \\
\hline
\end{tabular}

Note. Same as Table 4, for NGC 6239.

Table 15

H II Region Properties: UGC 5288

\begin{tabular}{|c|c|c|c|c|c|c|c|c|c|}
\hline $\begin{array}{l}\text { ID } \\
(1)\end{array}$ & $\begin{array}{l}\text { R.A. } \\
(2)\end{array}$ & $\begin{array}{l}\text { Decl. } \\
(3)\end{array}$ & $\begin{array}{l}R / R_{25} \\
\quad(4)\end{array}$ & $\begin{array}{c}R_{\text {proj }} \\
(5)\end{array}$ & $\begin{array}{c}F_{\mathrm{H} \alpha} \\
(6)\end{array}$ & $\begin{array}{l}m_{R} \\
(7) \\
\end{array}$ & $\begin{array}{c}{[\mathrm{O} \mathrm{II}]} \\
(8)\end{array}$ & $\begin{array}{c}{[\mathrm{O} \text { III }]} \\
(9)\end{array}$ & $\begin{array}{c}12+\log (\mathrm{O} / \mathrm{H}) \\
(10)\end{array}$ \\
\hline 4 & 095110.3 & +074917.4 & 2.3 & 3.07 & $0.19 \pm .02$ & $>24.57 \pm .34$ & 323 & 336 & 8.08 \\
\hline 1 & 095115.2 & +075035.9 & 1.4 & 1.93 & $0.22 \pm .02$ & $>24.57 \pm .34$ & 286 & 403 & 8.08 \\
\hline 12 & 095115.2 & +074942.0 & 0.7 & 0.88 & $0.95 \pm .11$ & $23.41 \pm .19$ & 441 & 198 & 8.18 \\
\hline 11 & 095115.9 & +074946.0 & 0.5 & 0.64 & $1.41 \pm .16$ & $21.93 \pm .08$ & 336 & 54 & 8.01 \\
\hline 10 & 095116.6 & +074950.7 & 0.4 & 0.48 & $25.57 \pm .06$ & $19.31 \pm .07$ & 270 & 461 & 8.09 \\
\hline
\end{tabular}

Note. Same as Table 4, for UGC 5288.

Table 16

H II Region Properties: UGC 9562

\begin{tabular}{|c|c|c|c|c|c|c|c|c|c|}
\hline $\begin{array}{l}\text { ID } \\
(1)\end{array}$ & $\begin{array}{l}\text { R.A. } \\
(2)\end{array}$ & $\begin{array}{c}\text { Decl. } \\
(3)\end{array}$ & $\begin{array}{c}R / R_{25} \\
\quad(4)\end{array}$ & $\begin{array}{c}R_{\text {proj }} \\
\text { (5) }\end{array}$ & $\begin{array}{c}F_{\mathrm{H} \alpha} \\
(6)\end{array}$ & $\begin{array}{l}m_{R} \\
(7)\end{array}$ & $\begin{array}{c}{[\mathrm{O} \mathrm{II}]} \\
(8)\end{array}$ & $\begin{array}{c}{[\mathrm{O} \text { III] }} \\
(9)\end{array}$ & $\begin{array}{c}12+\log (\mathrm{O} / \mathrm{H}) \\
(10)\end{array}$ \\
\hline 7 & 145112.7 & +353158.9 & 1.2 & 4.55 & $0.74 \pm .08$ & $22.97 \pm .31$ & 297 & 529 & 8.19 \\
\hline 12 & 145113.5 & +353158.2 & 1.1 & 4.12 & $0.44 \pm .05$ & $>23.17 \pm .36$ & 448 & 278 & 8.24 \\
\hline 17 & 145115.2 & +353258.0 & 0.8 & 3.18 & $1.24 \pm .14$ & $21.86 \pm .13$ & 396 & 200 & 8.11 \\
\hline 14 & 145113.9 & +353217.5 & 0.5 & 1.80 & $9.13 \pm .09$ & $19.99 \pm .07$ & 572 & 276 & 8.35 \\
\hline
\end{tabular}

Note. Same as Table 4, for UGC 9562.

over which it is useful. It is apparent from the $[\mathrm{N} \mathrm{II}] \lambda 6583 / \mathrm{H} \alpha$ ratios given in Tables 1 and 3 that most of the outlying and central $\mathrm{H}$ II regions lie on the upper branch of the R23 relation, except for a few cases which we discuss below.

We do not have any measurements of the $\mathrm{N} 2$ index for the outlying HII regions in NGC 2146 and therefore cannot definitively place them on either branch. Furthermore, the integrated [N II] $\lambda 6583 / \mathrm{H} \alpha$ ratios of NGC 3239 and the average $\left[\mathrm{N}_{\mathrm{II}}\right] \lambda 6583 / \mathrm{H} \alpha$ ratio of the $\mathrm{H}$ II regions in UGC 5288 put both galaxies in the ambiguous region of the N2 index. Fortunately, for UGC 5288 and NGC 3239, there are published values of the $\log [\mathrm{N} \mathrm{II}] /[\mathrm{OII}]$ that allow us to clear the $\mathrm{N} 2$ index ambiguity in the central regions. The integrated spectroscopy of the inner $R=3 \mathrm{kpc}\left(\sim 0.5 \times R_{25}\right)$ of NGC 3239 indicates a log $\left[\mathrm{N}_{\mathrm{II}}\right] /\left[\mathrm{O}_{\mathrm{II}}\right]$ of -1.05 (Moustakas \& Kennicutt 2006). UGC 5288 has an average $\log$ [N II]/[O II] of -1.16 within $R_{25}$ (van Zee \& Haynes 2006). Therefore, the inner H II regions of both galaxies lie on the lower branch of the $\mathrm{R} 23$ relation.

The outlying H II regions of NGC 3239 and UGC 5288 have $\mathrm{N} 2$ indices in the ambiguous region as well. In the case of UGC 5288, the upper branch M91 R23 values for its outer $\mathrm{H}$ II regions would put them at super-solar oxygen abundance, which is highly unlikely given that it is a dwarf galaxy and its central regions have an average oxygen abundance of 0.1 solar. We therefore assume that these outlying $\mathrm{H}$ II regions are on the lower branch of the R23 relation along with their more central counterparts. In the case of NGC 3239, the situation is less clear, since both upper- and lower-branch values of the oxygen abundance are near the R23 turnover region. We cannot make any assumptions for the outlying $\mathrm{H}$ II regions in this galaxy and therefore report both upper- and lower-branch values.

\section{RESULTS: THE DISTRIBUTION OF OXYGEN IN 13 H I ROGUES}

Here, we report radial oxygen abundance distributions for our sample of $13 \mathrm{H}$ I Rogues with Gemini GMOS-N spectral data. In this section, we consider each galaxy separately and comment on its environment (level of interaction), H I morphology, and the distribution of SF. For reference, H I column density contours, where available, are shown in Figure 1 for nine of the thirteen galaxies discussed here. The H I maps for those galaxies for which we were not able to construct our own zeroth moment maps are available for viewing in the H I Rogues Gallery (Hibbard et al. 2001).

Figure 2 shows the M91 R23 oxygen abundance, $12+\log$ $(\mathrm{O} / \mathrm{H})$, versus the galactocentric projected distances (in $R / R_{25}$ ) for the H II regions in each galaxy. McGaugh (1991) reports several different values of systematic error associated with his strong-line abundance calibration for zero-age $\mathrm{H}$ II regions, depending on the resultant oxygen abundance. He finds the systematic errors to be $\sim 0.1 \mathrm{dex}, 0.05 \mathrm{dex}$, and $0.20 \mathrm{dex}$ on the upper branch, lower branch, and within 0.1 dex of the turnover, respectively. Additional sources of error come from age effects, which can change the ionization parameter and shape of the ionizing spectrum (Stasińska \& Leitherer 1996), 

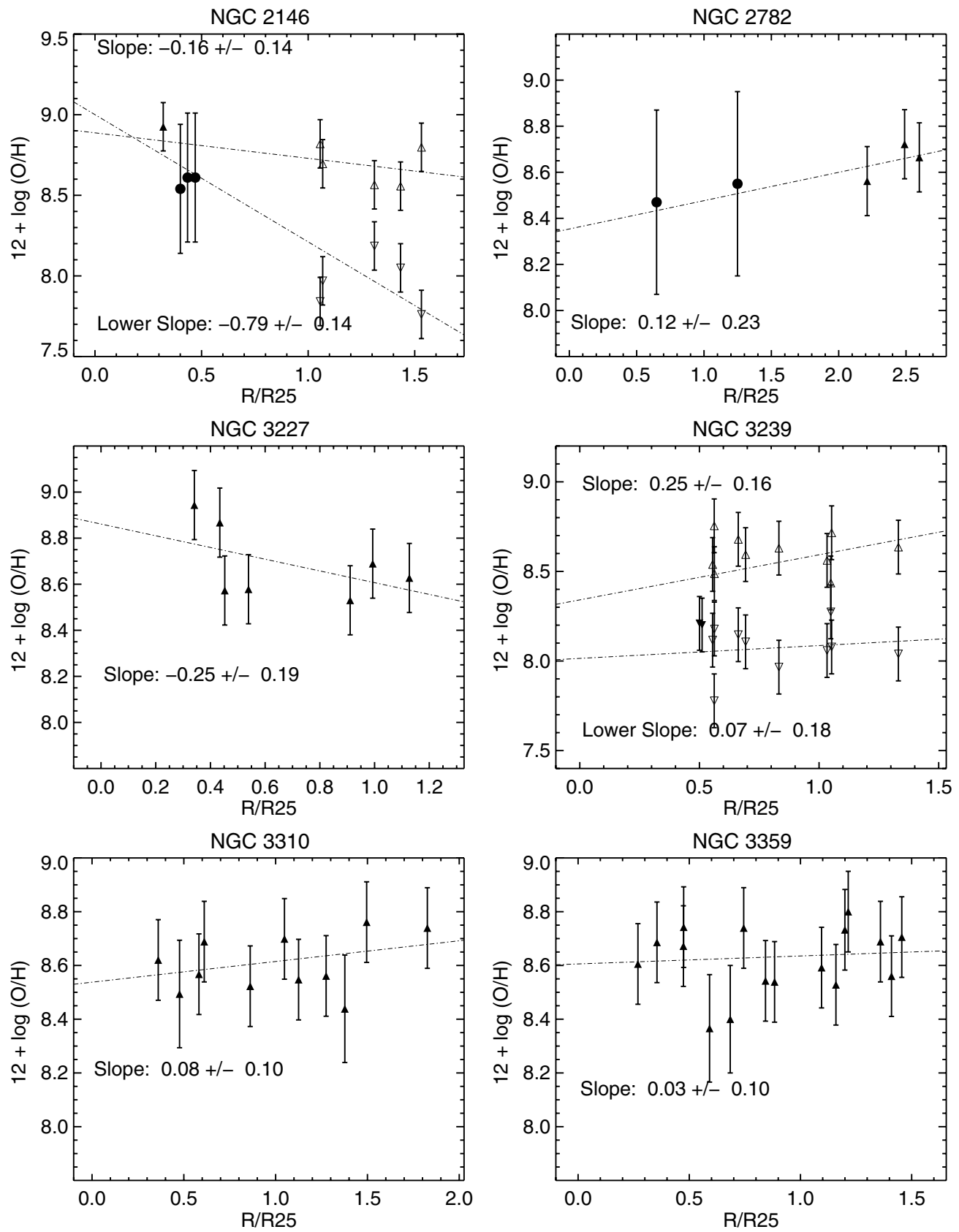

Figure 2. Radial oxygen abundance gradients, all in terms of $R / R_{25}$. The errors for the strong-line abundances range between 0.15 and 0.2 dex; the higher value is for abundances near the R23 turnover, $8.3<12+\log <8.5(\mathrm{O} / \mathrm{H})$. The dash-dotted line represents a linear least-squares fit to the abundance data, the slope (and its associated $\sigma$ ) of which is given on the plot. In two cases where we were unable to break the R23 degeneracy, NGC 2146 and NGC 3239 , we plot fits to both upper (upward-facing, open triangles) and lower (downward-facing, open triangles) branch values. In all cases except these two, we also put the gradients on the same $y$-axis for ease of comparison. The filled circles with larger error bars on the plots of NGC 2146 and NGC 2782 show PP04-derived oxygen abundances for reference. We could determine only PP04 abundances for these few H II regions that fell on the very edge of the GMOS slit masks.

and geometrical effects, which give rise to a large dispersion in ionization parameters (Ercolano et al. 2007). The overall impact of these effects increases the systematic errors of the M91 R23 calibration to $0.1-0.3$ dex, on average, regardless of branch, in the worst-case scenario. We therefore adopt $0.15 \mathrm{dex}$ as an average systematic error in our abundance measurements, unless a value is within 0.1 dex of the turnover, where it is then estimated to be 0.2 dex.

The dash-dotted line on each plot represents an errorweighted linear least-squares fit to the data. For reference, the solar oxygen abundance used in the M91 grid is $12+\log$ $(\mathrm{O} / \mathrm{H})_{\odot}=8.92$, and the turnover of the R23 relation occurs at $12+\log (\mathrm{O} / \mathrm{H})=8.35($ McGaugh 1991). In the cases of NGC 2146 and NGC 3239, we have not been able to break the degeneracy of the $\mathrm{R} 23$ relation for the majority of the $\mathrm{H}$ II regions and plot both upper-branch and lower-branch R23 abundances. On the gradients of NGC 2146 and NGC 2782, we show oxygen abundance values for a few $\mathrm{H}$ II regions derived from the PP04 calibration. We use different symbols for these abundances and larger error bars ( 0.4 dex) and caution against a direct comparison with the R23 abundances since the different calibrations are systematically offset from each other. We show these few points merely as a rough reference point where no other data are available. 

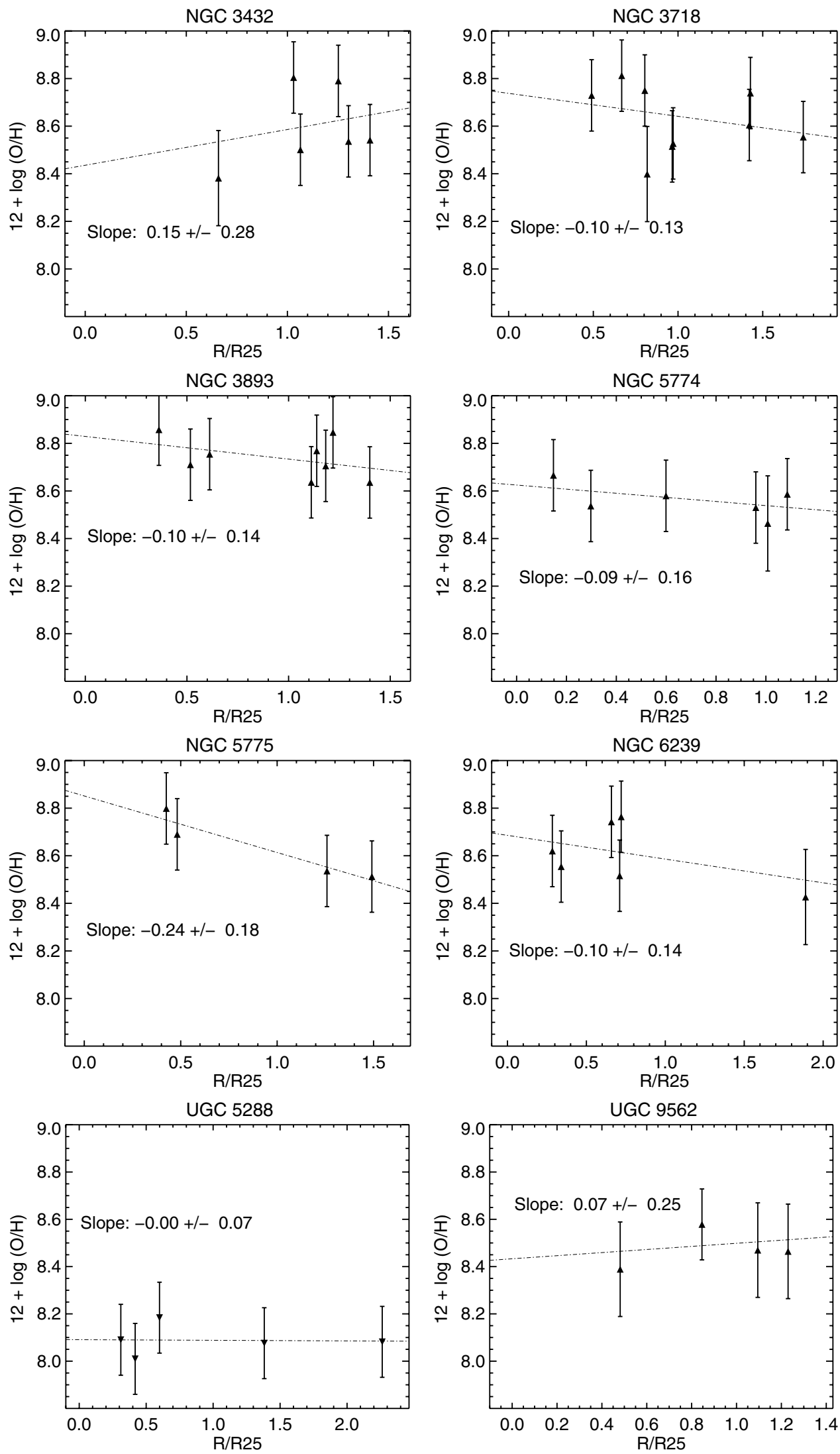

Figure 2. (Continued)

Examining dispersion in measurements relative to sample size, Zaritsky et al. (1994) find that five Hil regions per galaxy should serve as a minimum on which to base reliable measurements of radial abundance gradients. For reference, we measure at least five $\mathrm{H}$ II regions per galaxy in all but NGC 5775 and UGC 9562. We measure the oxygen abundances of only 
four $\mathrm{H}$ II regions in these galaxies. The range of projected radial distances over which we measure oxygen abundance gradients is generally between 0.4 and $1.5 R / R_{25}$, though several gradients extend beyond $2 R / R_{25}$. Tables 4 through 16 provide all of the relevant data on the $\mathrm{H}$ II regions for which we determine abundances in every galaxy: R.A. and declination, $R / R_{25}$, the projected distance from the nearest galaxy center in $\mathrm{kpc}$, $\mathrm{H} \alpha$ fluxes, and $R$-band magnitudes from MDM narrowband images, oxygen emission-line fluxes relative to $\mathrm{H} \beta=100$, and the oxygen abundance (upper- and lower-branch values, where appropriate).

1. NGC 2146 is classified in the $H_{\text {I }}$ Rogues catalog as a minor merger and has $\mathrm{H}$ i streams extending to the north and south out to six Holmberg radii (Taramopoulos et al. 2001). These streams are thought to be due to a tidal interaction between NGC 2146 and an unseen low surface brightness companion approximately $1 \mathrm{Gyr}$ ago. The outlying $\mathrm{H}$ II regions in this system are embedded within the H I of this system and are within the $(3-10) \times 10^{19} \mathrm{~cm}^{-2}$ column density contours. The total H I mass of NGC 2146 is $2.1 \times 10^{10} \mathcal{M}_{\odot}$, with $4.2 \times$ $10^{9} \mathcal{M}_{\odot}$ coming from within $R_{25}$ and $1.7 \times 10^{10} \mathcal{M}_{\odot}$ coming from the extended component. All nine $\mathrm{H}$ iI regions in NGC 2146 appear to lie on a single extended spiral arm that begins just to the southeast of the central burst of SF, turns north, and then ends at the outlying $\mathrm{H}$ II regions to the southwest of the galaxy center. Regions 12, 13, and 14 , all centrally located, lie at the edge of the CCD in the GMOS observations, and we therefore measure the oxygen abundance of these HiI regions using only the PP04 N2 index calibration (shown as filled circles in Figure 2). The dual-valued oxygen abundance for this galaxy complicates the interpretation of the gradient. If the outlying $\mathrm{H}$ II regions lie on the upper branch, then we find little to no abundance gradient $\left(-0.16\right.$ dex per $\left.R / R_{25}\right)$ for this galaxy. However, if the outlying $\mathrm{HII}$ regions have lower-branch oxygen abundances, then we find a very steeply declining gradient $\left(-0.79\right.$ dex per $\left.R / R_{25}\right)$ at large galactocentric radii.

2. NGC $\mathbf{2 7 8 2}$ is classified as a minor merger in the $\mathrm{HI}_{\mathrm{I}}$ Rogues catalog, with a one-sided H I tail on the opposite side of an extension of optical emission. The outlying $\mathrm{H}_{\mathrm{II}}$ regions are located within this $\mathrm{H}_{\mathrm{I}}$ tail, with column densities between $10^{19}$ and $10^{20} \mathrm{~cm}^{-2}$. The tail contains $3.4 \times 10^{9} \mathcal{M}_{\odot}$ or $\sim 80 \%$ of the galaxy's H I mass and is noted to consist of $\sim 10$ dwarf galaxy-sized clumps of $\sim 10^{8} \mathcal{M}_{\odot}$. The basic properties of NGC 2782 can be reproduced with the collision of two disk galaxies with a 1:4 mass ratio (Smith 1994). The three HII regions in the extended tidal tail of NGC 2782 (regions 1, 2, and 3 ) have the same oxygen abundance as the two H II regions closer to the center of the galaxy (regions 4 and 6), of $12+\log (\mathrm{O} / \mathrm{H}) \sim 8.6$. Regions 4 and 6 lie on the edge of the GMOS CCD, and we are able to use only the N2 index of PP04 to get rough estimates of their oxygen abundances, which are roughly consistent with the R23 values of Moustakas \& Kennicutt (2006) from the central nebular SDSS spectrum that give $12+\log (\mathrm{O} / \mathrm{H})=8.80$.

3. NGC 3226/7 is classified as an interacting double system in which only one of the galaxies is gas-rich in the H I Rogues catalog. NGC 3227 is the gas-rich galaxy and also a nearby Seyfert (Mundell 2001). H I tails extend $30 \mathrm{kpc}$ to the south and $70 \mathrm{kpc}$ to the north, beyond the region shown in our optical images. The total H I mass of the system is $2.0 \times$ $10^{9} \mathcal{M}_{\odot}$, with $\sim 1.0 \times 10^{9} \mathcal{M}_{\odot}$ of this mass located beyond
$R_{25}$. H II regions 24 and 25 are found within a cloud of $\mathrm{H}_{\mathrm{I}}$ that is thought to be a dwarf galaxy, while the outermost H II regions are found in outer lower density gas with column densities of a few $\times 10^{19} \mathrm{~cm}^{-2}$.

$\mathrm{H}$ II regions 10 and 12 are associated with the early-type galaxy NGC 3226, while the rest of the HiI regions in this system appear to be associated with NGC 3227. $R_{25}$ for this particular system was very difficult to obtain, given the overlap of the isophotes of the two merging galaxies. Instead of fitting $R_{25}$ for each galaxy, we fit $R_{25}$ for both galaxies together, which somewhat downplays the extended $\mathrm{SF}$ in this system. In a by-eye fit of $R_{25}$ to NGC 3227 alone, region 5 appears to lie at $R / R_{25}$ closer to 1.5 , and regions 24 and 25 are probably both further outside of $R_{25}$. Nonetheless, within the uncertainties, the outlying H II regions of this system appear to be about as oxygen-rich as those closer to the center of the system.

4. NGC 3239 is classified as an interacting double in which both of the galaxies are $\mathrm{H}$ I-rich with two $\mathrm{H}$ I tails according to the H I Rogues catalog (Iyer et al. 2001). In the direction of the extended arm of HiI regions shown in our optical images, the gas does not extend to a significantly larger radius, though there is a large $\mathrm{H}_{\mathrm{I}}$ feature on the opposite side of the galaxy. The outermost $\mathrm{H}$ II regions lie in gas that has a column density of $10^{20} \mathrm{~cm}^{-2}$. The H I mass of this galaxy is $1.2 \times 10^{9} \mathcal{M}_{\odot}$ (Huchtmeier \& Richter 1989), about $50 \%$ of which lies beyond $R_{25}$. Because the N2 indices for this galaxy lie in the ambiguous region (i.e., putting their resultant oxygen abundances near the turnover region of R23) we are unable to constrain the true abundance gradient. If we make the assumption that all outlying $\mathrm{H}$ II regions are either upper or lower branch, then the resultant abundance gradient is flat regardless of which branch is chosen. However, one can imagine a mixture of upper- and lower-branch oxygen abundances that would result in either increasing or decreasing gradients as well.

5. NGC 3310 is classified as a minor merger in the $H_{\text {I }}$ Rogues catalog. The gas disk of the main galaxy shows signs of perturbation from its high velocity dispersion and tails that extend $23 \mathrm{kpc}$ to the north and $51 \mathrm{kpc}$ to the south of the galaxy (Kregel \& Sancisi 2001). The Hi mass of the entire system is $5.2 \times 10^{9} \mathcal{M}_{\odot}$, with 4.2 and $5.0 \times 10^{8} \mathcal{M}_{\odot}$ in the northern and southern tails, respectively. Only the northern tail was imaged in $\mathrm{H} \alpha$ and contains the $\mathrm{H}$ II regions presented here. The peak column densities in the northern tail are $10^{20}-10^{21} \mathrm{~cm}^{-2}$. Most of the $11 \mathrm{H}$ II regions in this galaxy lie in an extended arm-like structure that extends to the northwest of NGC 3310, and all have the same oxygen enrichment of $12+\log (\mathrm{O} / \mathrm{H}) \sim 8.6$.

6. NGC 3359 is categorized as a galaxy with a detached H I cloud in the Hi Rogues catalog, but from the deeper contours it appears that the cloud may be a further extension of the extended H I spiral arm to the north of the galaxy (Boonyasait et al. 2001). The $\mathrm{H}$ in regions we study here are located within the extended gaseous envelope of the galaxy, but not this far extended arm. NGC 3359 contains $4.5 \times$ $10^{9} \mathcal{M}_{\odot}$ in neutral hydrogen gas, and we estimate that about half of it is contained within $R_{25}$.

The $16 \mathrm{H}$ II regions we consider in NGC 3359 all have similar oxygen abundances near $12+\log (\mathrm{O} / \mathrm{H}) \sim 8.6$. Martin \& Roy (1995) report oxygen abundances for 77 H II regions within $R_{25}$ for this galaxy and find a steeply decreasing oxygen gradient in the centermost part of the 
galaxy, out to $\sim 0.35 R_{25}$, and then a flat oxygen abundance gradient that levels off at $12+\log (\mathrm{O} / \mathrm{H})=8.6$ from 0.35 to $0.9 R_{25}$. Our results are consistent with this flat gradient and indicate that it extends to even larger radii, out to $1.5 R_{25}$.

7. NGC 3432 is noted as a minor merger in the Hi Rogues catalog. It has a strong warp and a companion to the southwest corresponding to the side with the most dominant warp (Swaters et al. 2002). The total $\mathrm{HI}$ mass of the system is $6.0 \times 10^{9} \mathcal{M}_{\odot}$, with $2.58 \times 10^{9} \mathcal{M}_{\odot}$ in the outer regions beyond $R_{25}$. The $\mathrm{H}$ II regions studied here are in the northern part of the galaxy's gaseous disk, with some of the outermost $\mathrm{H}$ II regions lying in gas at $4 \times 10^{20} \mathrm{~cm}^{-2}$. NGC 3432 appears to have a flat oxygen abundance gradient (within the large error) with a median value of $12+\log$ $(\mathrm{O} / \mathrm{H}) \sim 8.5$.

8. NGC 3718 is a galaxy located in the outer regions of the Ursa Major Cluster with a two-sided extreme H I warp (Verheijen \& Sancisi 2001). It has a total mass of $10^{10} \mathcal{M}_{\odot}$ in neutral hydrogen and an FWHM HI line width of $465 \mathrm{~km} \mathrm{~s}^{-1}$. The H II regions studied here are generally not located in the highest column density gas along the warp, although that is where much of the new SF appears to be concentrated. All nine $\mathrm{H}$ II regions presented here have abundances consistent with the mean of $12+\log (\mathrm{O} / \mathrm{H})$ $\sim 8.6$.

9. NGC 3893 is classified as an M51-type minor merger and is located in the Ursa Major Cluster (Verheijen \& Sancisi 2001). The interacting companion (NGC3896) and NGC3893 appear to be embedded in a common envelope of Hi. The HiI regions studied here are embedded in high column density gas in the inner part of the large envelope and appear to trace an extended, low surface brightness spiral arm. The H I mass of this system is $6 \times 10^{9} \mathcal{M}_{\odot}$, about half of which is located within $R_{25}$. All eight of the H II regions we study in NGC 3893 have similar oxygen abundances, near the mean of $\sim 8.75$.

10. NGC 5774/5 is classified in the Hi Rogues catalog as an interacting double with no tails and an $\mathrm{H}$ i bridge joining the two gas-rich galaxies. H II regions in both galaxies and in the $\mathrm{H}$ I bridge were targeted in this study. The NGC 5774/5 system belongs to a small group of at least six galaxies and the gas in the bridge between the galaxies is thought to be transferring gas from NGC 5774 to NGC 5775 (Irwin 1994). The Hi masses of the galaxies are 5.4 and $9.1 \times 10^{9} \mathcal{M}_{\odot}$, respectively. The oxygen abundances of the $\mathrm{H}$ II regions in this system are all close to an average value of $12+\log$ $(\mathrm{O} / \mathrm{H}) \sim 8.7$.

11. NGC 6239 is a minor merger in the Hi Rogues catalog. Both galaxies appear to be gas-rich, although we imaged only the primary galaxy in $\mathrm{H} \alpha$. The one outlying $\mathrm{H}_{\text {II }}$ region in this galaxy is in the northern $\mathrm{H}_{\mathrm{I}}$ tail with an H I column density of $5 \times 10^{19} \mathrm{~cm}^{-2}$. The H I mass of this galaxy is $7 \times 10^{9} \mathcal{M}_{\odot}$ (Springob et al. 2005). The radial oxygen abundance gradient is consistent with being flat over $\sim 15 \mathrm{kpc}$.

12. UGC 5288 is a blue compact dwarf galaxy with an extended $\mathrm{H}$ I envelope and no sign of interaction in the gas disk. Its optical and $\mathrm{H}$ i properties are qualitatively similar to those of NGC 2915 (see Werk et al. 2010a). The H II regions probed in the outer gaseous regions of this galaxy are found in gas with column densities as low as $2 \times 10^{20} \mathrm{~cm}^{-2}$. The total H I mass of this galaxy is $2 \times 10^{8} \mathcal{M}_{\odot}$ (Wong et al.
2006), only $10 \%$ of which is within $R_{25}$. The radial oxygen abundance gradient for UGC 5288 is flat.

13. UGC 9562 (also known as II Zw 71) is classified as an interacting double (the companion is UGC 9560/II Zw 70) or a polar ring galaxy. Both galaxies are gas-rich, with a bridge joining the two galaxies and a tail on the opposite side of the bridge linked to UGC 9562 (Cox et al. 2001). All of the Hil regions we study here are close to UGC 9562 which contains $8.2 \times 10^{8} \mathcal{M}_{\odot}$ in $\mathrm{H}$ I. The oxygen abundance gradient in UGC 9562 is flat, with an average oxygen abundance near the turnover of R23.

Excluding the two ambiguous cases (NGC 2146 and NGC 3239), our derived abundance gradients range from $+0.25 \mathrm{dex} / R_{25}$ to $-0.25 \mathrm{dex} / R_{25}$. We note that our data generally do not apply to nuclear oxygen abundance gradients between 0 and $\sim 0.4 R / R_{25}$, which may very well be declining at different rates. Furthermore, we derived these gradients using projected galactocentric distances. Given uniformly shallow, if not flat, radial abundance gradients, correcting for inclination angle would have little impact on the overall results. The errorweighted mean of the radial abundance gradients (excluding the two ambiguous cases), normalized to $R / R_{25}$, is $-0.02 \pm 0.18$. These galaxies have radial oxygen abundance gradients (beyond $0.4 R / R_{25}$ ) consistent with being flat within $1 \sigma$ over an average of $\sim 15 \mathrm{kpc}$, where typical $\sigma_{\text {slope }}=0.15$ dex. Additionally, NGC 3239 is consistent with these results, regardless of branch choice. If the outlying H II regions of NGC 2146 lie on the upper branch as they do in the vast majority of our sample of galaxies, then NGC 2146, too, is consistent with having a flat abundance gradient. Alternatively, NGC 2146 may be the only galaxy in our sample that has a significantly declining radial abundance gradient of $-0.79 \mathrm{dex} / R_{25}$, corresponding to $-0.035 \mathrm{dex} \mathrm{kpc}{ }^{-1}$. However, this gradient is still somewhat shallow compared to those of typical spiral galaxies, $-0.07 \mathrm{dex} \mathrm{kpc}^{-1}$ (Zaritsky et al. 1994; see Section 6.1).

\subsection{Gas Densities in the Outer Disks: Low-density Star Formation}

The Hi gas densities at the locations of the outlying $\mathrm{H}$ II regions range from $(3-500) \times 10^{19} \mathrm{H} \mathrm{I}$ atoms $\mathrm{cm}^{-2}$, corresponding to a range in gas surface density of $(0.25-80) M_{\odot} \mathrm{pc}^{-2}$. The median value, which is typical of most of the gas column densities at the outlying $\mathrm{H}$ II region locations, is $4 \times 10^{20} \mathrm{~cm}^{-2}$ or $\sim 3 M_{\odot} \mathrm{pc}^{-2}$. This gas surface density is at the traditional "threshold" level for SF, usually cited between 2 and $8 M_{\odot} \mathrm{pc}^{-2}$ (Schaye 2004). Thus, we are truly examining SF at low average gas surface densities, in the same outer-disk regime explored by Fumagalli \& Gavazzi (2008) and Bigiel et al. (2010). The outlying $\mathrm{H}$ II regions presented here cover a range of $\mathrm{H} \alpha$ luminosities, from $10^{35.5}$ to $10^{37.5} \mathrm{erg} \mathrm{s}^{-1}$, corresponding to SFRs that range from $2 \times 10^{-4}$ to $2 \times 10^{-6} M_{\odot} \mathrm{yr}^{-1}$. Most of the outlying $\mathrm{H}$ II regions are isolated within surface areas of roughly a $\mathrm{kpc}^{2}$, so these SFRs can be viewed roughly as SFR surface densities in units of $M_{\odot} \mathrm{yr}^{-1} \mathrm{kpc}^{-2}$. These values of SFRs and $\mathrm{H}$ I gas surface densities place the outlying $\mathrm{H}$ II regions in the same, or lower, "downturn" portion of the Kennicutt-Schmidt law (Wyder et al. 2009), as expected. One caveat to keep in mind is that these stars may be forming in places where the local gas density is higher than the average surrounding gas densities, on a size scale which would be unresolved in the H I synthesis maps. We will present a full analysis of the relationship between the atomic hydrogen content and SF activity in the gaseous outskirts of Hi Rogues in a future paper. 


\section{DISCUSSION: THE LACK OF SIGNIFICANT ABUNDANCE GRADIENTS AT LARGE GALACTOCENTRIC RADII}

The $13 \mathrm{H}$ I Rogue galaxies in our sample are diverse, in terms of both their $\mathrm{H}_{\mathrm{I}}$ and optical properties. Their total baryonic masses range from dwarf galaxies with $M<10^{9} \mathcal{M}_{\odot}$ (UGC 5288) to massive spiral galaxies with $M \sim 10^{11} \mathcal{M}_{\odot}$ (NGC 2146). Their H I morphologies range from very extended, undisturbed $\mathrm{H}$ I envelopes, to warped $\mathrm{H}$ I distributions, to large tidal tails, representing a wide range of galaxy interactions; and, their SFRs range from $0.006 \mathcal{M}_{\odot} \mathrm{yr}^{-1}$ to $3 \mathcal{M}_{\odot} \mathrm{yr}^{-1}$. Our results show that the outer gas of disturbed, interacting, or extendedgas disk galaxies is oxygen-enriched. Predominantly flat radial oxygen abundance gradients in this wide variety of galaxies with varying degrees of interaction and SF provide compelling evidence of uniform metal distribution across extended gaseous features independent of large-scale galaxy properties and individual accretion histories. In this section, we present an analysis of the oxygen yields of all the Rogue galaxies, their positions on the mass-metallicity relation, and discuss the implications of flat oxygen abundance gradients out to large galactocentric radii.

\subsection{Comparison with Previous Work}

First, we discuss previous work on the subject of radial abundance distributions and attempt to reconcile it with the flat gradients observed here. Declining radial abundance gradients, often out to, and/or slightly beyond $R_{25}$, are commonly observed in spiral galaxies (Oey \& Kennicutt 1993; Zaritsky et al. 1994; Kennicutt et al. 2003). The broadest study to date is that of Zaritsky et al. (1994). They present metallicity gradients between 0.1 and $1.0 R / R_{25}$ using at least five $\mathrm{H}$ II regions in each of 39 local spiral galaxies with a wide range of morphologies (Sab-Sm; seven galaxies have bars) and luminosities $\left(-21<M_{B}<-17\right)$. Because the scatter in oxygen abundance at a given galactic radius ( 0.4 dex at most) for each galaxy is much smaller than the mean abundance range for their entire sample (8.34-9.31), they conclude that these gradients are determined globally, rather than set by local conditions (i.e., the local SFR and gas fraction). Zaritsky et al. (1994) find a wide range of slopes in the 39 radial oxygen distributions, from flat to very steeply declining, at $-1.45 \mathrm{dex} / R_{25}$ corresponding to $-0.231 \mathrm{dex} \mathrm{kpc}^{-1}$. Their average slope is -0.59 $\mathrm{dex} / R_{25}$ or $-0.07 \mathrm{dex} \mathrm{kpc}^{-1}$. Therefore, we do not see declining abundance gradients similar to those of Zaritsky et al. (1994) where our average gradient is $-0.02 \pm 0.18 \mathrm{dex} / R_{25}$ or $-0.001 \mathrm{dex} \mathrm{kpc}^{-1}$. Furthermore, our data rule out decreasing radial oxygen abundance gradients steeper than -0.30 dex over $15 \mathrm{kpc}\left(-0.02 \mathrm{dex} \mathrm{kpc}{ }^{-1}\right)$ in these outer regions.

Several studies of metallicity gradients in the outer parts of relatively quiescent spiral galaxies have found a flattening beyond $0.5 R / R_{25}$ (Martin \& Roy 1995; Bresolin et al. 2009). Similarly, studies of low surface brightness disk galaxies (de Blok \& van der Hulst 1998) and dwarf galaxies (Croxall et al. 2009) are suggestive of flat oxygen abundance gradients out to $R_{25}$. And, the metallicity gradients of interacting pairs appear to be consistently flatter than those of spiral galaxies (Kewley et al. 2010). Yet, this study is the first to measure radial oxygen abundance gradients in the outer regions of a modest sample of galaxies beyond $R_{25}$. The majority of our Galaxies are interacting, which may partially explain a flattening in the abundance gradients (Rupke et al. 2010; Kewley et al. 2010). Given the wide range of systems (including minor mergers and warps) over which we measure flat gradients, our results suggest that galaxy interactions are not the sole explanation.

\subsection{Relationship to the Mass-Metallicity Relation}

There is a fundamental, global relation between galaxy mass and metallicity in the sense that lower mass galaxies have lower overall heavy-element content. Larson (1974) first predicted that the average stellar metal abundance in a galaxy would depend on its mass owing to more significant gas loss from the energy supplied by supernovae in lower mass galaxies. His notion turned out to fit well with observations of nebular oxygen abundances of irregular galaxies several years later by Lequeux et al. (1979) and has become known as the mass-metallicity relation for galaxies in the 36 years since (Skillman et al. 1989; Tremonti et al. 2004). This relation is often parameterized in terms of effective yields and total (gas + stellar) mass to elucidate its origin, where effective yield measures how much a galaxy's metallicity deviates from its "closed-box" value, given a certain gas mass fraction (Dalcanton 2007 provides a more detailed, clear explanation). Systematically, low effective yields of dwarf galaxies have been taken as evidence that low-mass galaxies have evolved less as "closed boxes" than their more massive counterparts (Tremonti et al. 2004).

Although supernova-driven metal expulsion is the most popular and classic explanation for the low effective yields of dwarf galaxies (Larson 1974; Tremonti et al. 2004), there are several issues that complicate the picture. Dalcanton (2007) shows, for instance, that metal loss itself does not have a strong dependence on galaxy mass and that any subsequent SF following an episode of mass loss quickly pushes the effective yield up to its closedbox value. In the calculations presented by Dalcanton (2007), low SF efficiencies in gas-rich dwarfs between episodes of mass loss are what keep their effective yields low. Dilution by infalling low-metallicity gas may also play some role, but alone cannot explain the mass-metallicity relation (Dalcanton 2007). An explanation offered by Tassis et al. (2008) is that the metals are not expelled via supernova-driven winds, but rather efficiently mixed via gravitational processes into the hot gas, where they are unobservable by standard ground-based methods. Coupled with low SF efficiencies of low-mass, gas-rich dwarfs, Tassis et al. (2008) can reproduce the observed mass-metallicity relation without including winds from supernova in their model. Finally, if low-mass galaxies preferentially do not make as many O-type stars as more massive galaxies (Meurer et al. 2009; Lee et al. 2009), as in the IGIMF theory, then their effective yields will appear to be low (Köppen et al. 2007).

We calculate the effective oxygen yield for each galaxy, $Z / \ln \left(\mu^{-1}\right)$, where $\mu$ is the gas fraction and $Z$ is the metallicity by mass, using the mean $\mathrm{H}$ II region oxygen abundance as a proxy for total metal content, the stellar masses listed in Table 1, and total gas masses computed from the H I masses given in Table 1 plus a $36 \%$ contribution from helium. We do not include molecular gas in these calculations, although it most certainly is present. Although the molecular-to-neutral gas content varies widely within and among galaxies (Bigiel et al. 2008; Leroy et al. 2008), the total galaxy molecular gas mass is rarely, if ever, larger than its neutral gas mass. If we assume that, on galaxy-wide scales, the molecular gas content scales to first order with the neutral gas content, then the effect on the overall mass-metallicity relation would be negligible.

Figure 3 (upper panel) plots the effective oxygen yield versus total baryonic mass for our Galaxies (filled circles; NGC 5774 and NGC 5775 we consider separately, though they 

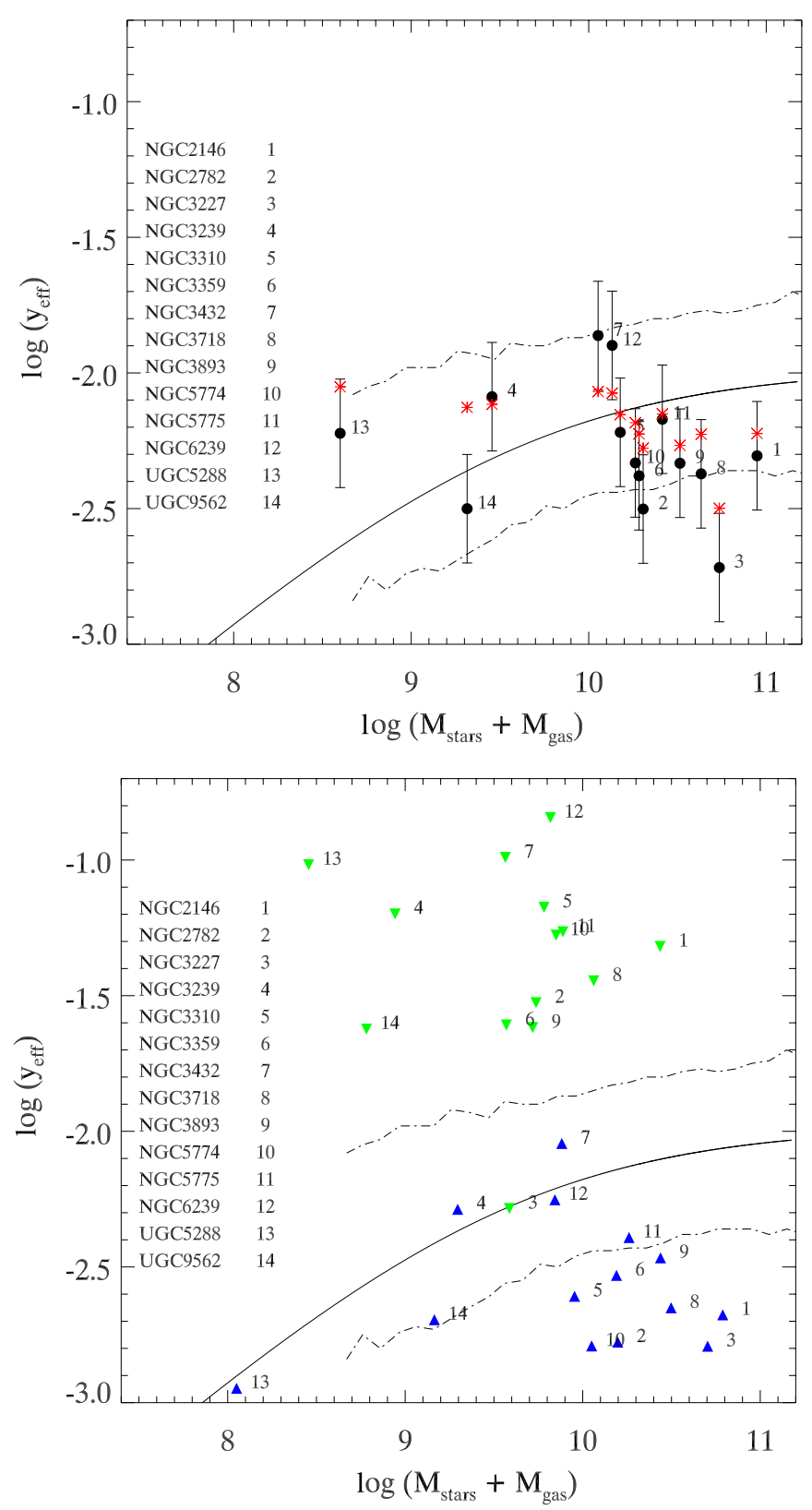

Figure 3. Top: the effective yield vs. the total baryonic (stellar plus gas) mass for the H II regions in every galaxy, shown as filled circles. We plot "expected" oxygen yields, determined from total stellar masses and a constant net oxygen yield, for each galaxy as red asterisks. Bottom: the "inner" and "outer" galaxy have been separated. The blue triangles show the inner $\left(R<R_{25}\right)$ galaxies, and the green triangles represent the outer $\left(R>R_{25}\right)$ regions. Each point is marked with a number that corresponds to a galaxy listed in the key on the left-hand side of the figure. The empirical relation in Tremonti et al. (2004), their Equation (6), is shown for reference (solid line), along with contours (dash-dotted lines) that enclose 95\% of the SDSS data presented in Tremonti et al. (2004).

are technically part of the same H I envelope) along with the empirical relation of Tremonti et al. (2004). To include both NGC 2146 and NGC 3239 in this figure, we have assumed upperbranch metallicities for the $\mathrm{H}$ II regions. While this assumption may not be valid, the points in the upper panel of Figure 3 do not change appreciably with branch assumption. With the exception of NGC 3227, every galaxy in our sample appears to lie along the empirical Tremonti et al. (2004) relation, within the $95 \%$ contours (dash-dotted lines). Tremonti et al. (2004) explain the decrease in metal yields with decreasing galaxy baryonic mass by invoking metal-rich supernova-driven winds that preferentially escape from smaller potential wells of lessmassive galaxies.

The red asterisks in Figure 3 (upper panel) directly above or below the filled-circle galaxy points mark an "expected" oxygen yield determined by using the galaxy's total stellar mass and assuming a net oxygen yield of 0.01 (average value from Maeder 1992; the mass of oxygen ejected by all stars per unit mass of matter locked up in stars). All galaxies, with the exception of UGC 9562, have yields consistent with those predicted from a rough estimate that simply accounts for all metals using the existing stellar population.

As was the case with NGC 2915, considered in Werk et al. (2010a), there is only a low level of ongoing and previous SF at the locations of the outermost $\mathrm{H}$ II regions. In this sense, the lack of radial oxygen abundance gradients beyond the optical "edges" of galaxy disks seems to necessarily imply some type of efficient metal transport. In the context of the mass-metallicity relationship, we can consider the yields for the outer and inner gaseous regions separately. We show this separation in Figure 3 (lower panel) with the assumption that $95 \%$ of the stellar mass is contained within $R_{25}$, and calculating the H I mass within $R_{25}$ from the $\mathrm{HI}_{\mathrm{I}} 21 \mathrm{~cm}$ synthesis data, when it was available to us. In the four cases that it was not, we estimated by eye the total H I mass within and outside of R25 from the H I column density maps available from the online the $\mathrm{H}_{\mathrm{I}}$ Rogues Gallery. The percentage of H I mass contained in $R_{25}$ varies from $10 \%$ to $60 \%$ for the galaxies.

First, keeping metallicity constant while lowering the stellar mass contribution to the effective yields relative to the gas mass contribution (outer $\mathrm{H}$ II regions) shifts the black points in Figure 3 (upper panel) to the left and up, shown as downwardfacing green triangles in Figure 3 (lower panel). Note that the green triangles for NGC 2146 and NGC 3239 move down by $0.7 \mathrm{dex}$ and $0.4 \mathrm{dex}$, respectively, if we instead assume lower-branch metallicities for their outer HII regions. This figure shows that the effective yields for the outer disk lie above their "closed-box" values. Conversely, the general trend of lowering the gas mass contribution relative to the stellar mass contribution while keeping metallicity constant (inner H II regions) has the effect of moving the points to the left and down, shown as blue triangles in Figure 3 (lower panel). For the most part, the effective yields for the inner disk fall below their "closed-box" values. Including molecular gas in this analysis would only make the effect more striking, since, in general, the fraction of molecular gas decreases with galactocentric radius (Leroy et al. 2008). Outer-galaxy oxygen abundances are generally more metal-enriched than their gas fractions otherwise suggest, while inner-galaxy oxygen abundances lie below what is expected based on these simple stellar-populationbased assumptions. This duality appears to underlie the flat radial abundance gradients of gas-rich, interacting galaxies and apparently isolated extended gaseous disk dwarf galaxies (UGC 5288 and NGC 2915; Werk et al. 2010b).

\subsection{Methods of Metal Transport}

As with the flat radial oxygen abundance gradient of NGC 2915, a number of physical mechanisms could be at work in various combinations to distribute metals in these Hi Rogue galaxies. Very generally, our results indicate that there is efficient metal mixing out to large galactocentric radii, facilitated by galaxy interactions. Nonetheless, there are a few galaxies in our sample that show no evidence of a recent merger or interaction, 
yet are still well mixed out to large radii. The rest of our sample represents a myriad of interaction types. If indeed interactions are the primary driver of the metal mixing seen here, then the mechanism by which it acts is independent of the interaction details.

The accretion of gas following a merger event or tidal interaction results in tidal torques that drive the gas to the system center (Barnes \& Hernquist 1996) and subsequently mix metals into the outer regions (Rupke et al. 2010). Other than tidal torques, metal mixing can be driven by a number of processes: magnetorotational instabilities (Sellwood \& Balbus 1999), infall of gas clouds (Santillán et al. 2007), self-gravity in conjunction with differential rotation (Wada \& Norman 1999), quasi-stationary spiral structure interacting with a central bar (Minchev \& Famaey 2010), viscous flows generated by gravitational instability or cloud-cloud collisions (Ferguson \& Clarke 2001), and thermal instability triggered self-gravitational angular momentum transport (McNally et al. 2009). In general, the sound speed, $\sim 10 \mathrm{~km} \mathrm{~s}^{-1}$, is a robust upper limit to the speed at which mixing occurs in cold, neutral gas. Measurements of velocity dispersions in the outer $\mathrm{H}$ I disks of galaxies are roughly consistent with this number, generally no less than $8 \mathrm{~km} \mathrm{~s}^{-1}$ (Tamburro et al. 2009). In some strongly interacting galaxies, the velocity dispersions may be higher due to these gravitational perturbations, up to $30 \mathrm{~km} \mathrm{~s}^{-1}$ in some rare cases (e.g., NGC 1533; Ryan-Weber et al. 2003). Furthermore, in the extended H I disk of NGC 2915, Elson et al. (2010) find evidence for radial flows of $5-17 \mathrm{~km} \mathrm{~s}^{-1}$ that may be driving central material outward into the extended disk. They note that these flows are consistent with the finding of higher-than-expected oxygen abundances in the outer gaseous disk, as observed by Werk et al. (2010a).

A simple calculation of mixing timescales, assuming that there is mixing at the sound speed over $15 \mathrm{kpc}$, yields $1.5 \mathrm{Gyr}$ as the lower limit to the timescale over which mixing occurs in our extended HI features. Yet, the metals generated by massive stars are generally returned to the ISM on much shorter timescales, on the order of 100 Myr (Tenorio-Tagle 1996). Even if the velocity dispersion in the cold gas is three times higher (30 $\mathrm{km} \mathrm{s}^{-1}$; the most extreme end) due to interactions, the lower limit to the cold-gas mixing timescale is $500 \mathrm{Myr}$. By assuming a net oxygen yield of 0.01 (Maeder 1992; the mass of oxygen ejected by all stars per unit mass of matter locked up in stars), we can calculate this required average outward metal "momentum" that would reproduce a flat oxygen distribution. Taking an average inner- $R_{25}$ SFR of $0.5 M_{\odot} \mathrm{yr}^{-1}$, and an average separation between inner and outer SF of $15 \mathrm{kpc}$, we find that there needs to be a constant outward movement of $0.0025 M_{\odot}$ of oxygen at $150 \mathrm{~km} \mathrm{~s}^{-1}$. We can repeat this calculation for the most compact galaxies in our sample (NGC 3239, UGC 5288, and UGC 9562), now taking an average inner- $R_{25}$ SFR of $0.15 M_{\odot} \mathrm{yr}^{-1}$ and an average separation between inner and outer SFs of $4 \mathrm{kpc}$. The corresponding "metal momentum" needed to wipe out any abundance gradient is $0.0007 M_{\odot}$ of oxygen at $40 \mathrm{~km} \mathrm{~s}^{-1}$. While metal mixing in cold neutral gas probably happens, it most likely does not happen on fast enough timescales to wipe out a metallicity gradient that would arise in a typical star-forming spiral galaxy from a radial dependence of the SFR (i.e., SF is concentrated in the central regions, therefore metals are concentrated in central regions). With the advent of high-resolution radio telescopes such as the EVLA and ALMA, future work will be able to address the kinematics and motions of cold gas in considerably more detail.
Given the fast mixing speeds required, we consider that the metal transport may be occurring predominantly in a hot gas component as proposed by Tassis et al. (2008) and others. Although the dominant driver of the mixing of metals in the hot-phase gas is unclear, suggested sources include diffusion, gravitational instabilities, cold accretion flows, and galaxy interactions (Kereš et al. 2005; Tassis et al. 2008). The recent finding of $\mathrm{O}$ VI absorption over the entire Milky Way disk, irrespective of circumstellar environments and spiral arms (Bowen et al. 2008), may provide some support for the idea that there can be efficient mixing in the hot gas phase of the ISM. Furthermore, the hot X-ray gas generated by metal-enriched supernova-driven blowouts (i.e., winds) is able to propagate to the outer $100 \mathrm{kpc}$ of a galaxy's halo (Strickland et al. 2004). Finally, the three-dimensional adaptive mesh refinement code of de Avillez \& Mac Low (2002) finds that inhomogeneities in the ISM can be erased over a couple hundred Myr (dependent on supernova rate) when gas cycles between the disk and the hot halo in a galactic fountain process. Whether or not enough metals can be transported in the hot gas and quickly cooled at large enough radii to reproduce the flat oxygen distribution seen in these 13 galaxies is unclear. However, our data generally support a scenario in which galaxy interactions induce efficient metal mixing in hot gas over the full scale of the galaxy.

Efficient metal mixing has important implications for the origin of the mass-metallicity relationship. Large-scale and universal metal outflow may not be the dominant cause of the low effective yields in some gas-rich dwarf galaxies as advanced by Tremonti et al. (2004) and others. Instead, these low-mass gas-rich galaxies on the whole would have fewer metals than expected from the simple nucleosynthetic yield because they are stirring those centrally generated metals throughout their gaseous outskirts. Coupled with the idea that SF is increasingly inefficient in these low-mass, gas-rich galaxies (Dalcanton 2007), mixing could account for lower effective yields in lower mass galaxies with no real need for large-scale metal blow out.

\section{SUMMARY AND CONCLUSIONS}

Using narrowband $\mathrm{H} \alpha$ and continuum $R$-band images from the MDM 2.4 m telescope, we have identified 13 "H i Rogues" with massive SF occurring beyond their optical radii. GMOS multi-slit optical spectroscopy targeting both inner and outlying $\mathrm{H}$ II regions in these 13 Rogue galaxies allowed for the measurement of strong-line oxygen abundances of $\sim 100 \mathrm{H}$ iI regions with projected galactocentric distances ranging from 0.3 to $2.5 R / R_{25}$. Despite the diversity of these 13 systems in terms of their optical and Hi morphologies, star-forming properties, and level of disruption, we find that all of them have flat radial oxygen abundance gradients from their central optical bodies to their outermost regions. In addition to the interacting systems with flat abundance gradients, we find several non-interacting, gas-rich galaxies that have flat oxygen abundance gradients out to large projected radii.

We find that efficient metal-mixing or pre-enrichment and subsequent mixing from recent interactions are the two most likely potential causes for the enrichment of the gas beyond $R_{25}$. Large-scale metal blowout then may not be the primary driver of the mass-metallicity relation for gas-rich or interacting galaxies. That metals are distributed throughout the extended gaseous regions of both interacting and non-interacting, strongly star-forming and quiescent, massive and dwarf galaxies has 
important implications for the origin of the mass-metallicity relation, and galaxy chemical evolution in general.

J.K.W. thanks members of her dissertation committee at the University of Michigan for valuable insights and suggestions that improved this final manuscript: Fred Adams, Lee Hartmann, Mario Mateo, and Sally Oey. Discussions with Eric Pelligrini and the entire University of Michigan FANG (Feedback Activity in Nearby Galaxies) research group were also very helpful for understanding the results of this work. J.K.W. gratefully acknowledges the generosity of the COS-halos group, specifically Xavier Prochaska, for permitting the Keck LRIS observations of several H I Rogues that were instrumental in breaking the R23 degeneracy. Observations at Lick went considerably more smoothly because of the aid of Michele Fumagalli and Robert da Silva-many thanks to both. The following individuals kindly provided their reduced $\mathrm{H}$ I data cubes for the interpretation of our results: Judith Irwin (NGC 5774/5), Carole Mundell (NGC 3227), Beverly Smith (NGC 2782), David Hogg and Mort Roberts (NGC 6239), Caroline Simpson (NGC 3239), Rob Swaters (NGC 3432), Marcel Clemens (NGC 3395; not used), and Marc Verheijen (NGC 3718 and NGC 3893). Finally, J.K.W. wishes to thank the very helpful support staff, both at MDM and Gemini Observatories. M.E.P. and J.K.W. acknowledge support for this work through NSF CAREER AST0904059, the Research Corporation, and support from the Luce Foundation. G.R.M. was partially supported by NASA LTSA grant NAG5-13083 for the work presented here.

Facilities: Gemini:Gillett, Hiltner, Keck:I, VLA, WSRT

\section{REFERENCES}

Barnes, D. G., et al. 2001, MNRAS, 322, 486

Barnes, J. E., \& Hernquist, L. 1996, ApJ, 471, 115

Bell, E. F., McIntosh, D. H., Katz, N., \& Weinberg, M. D. 2003, ApJS, 149, 289

Bigiel, F., Leroy, A., Walter, F., Blitz, L., Brinks, E., de Blok, W. J. G., \& Madore, B. 2010, AJ, 140, 1194

Bigiel, F., Leroy, A., Walter, F., Brinks, E., de Blok, W. J. G., Madore, B., \& Thornley, M. D. 2008, AJ, 136, 2846

Boonyasait, V., Gottesman, S. T., \& Broeils, A. H. 2001, in ASP Conf. Ser. 240, Gas and Galaxy Evolution, ed. J. E. Hibbard, M. Rupen, \& J. H. van Gorkom (San Francisco, CA: ASP), 857

Bowen, D. V., et al. 2008, ApJS, 176, 59

Bresolin, F., Kennicutt, R. C., Jr., \& Garnett, D. R. 1999, ApJ, 510, 104

Bresolin, F., Ryan-Weber, E., Kennicutt, R. C., \& Goddard, Q. 2009, ApJ, 695, 580

Calzetti, D. 2008, in ASP Conf. Ser. 390, Pathways Through An Eclectic Universe, ed. J. H. Knapen, T. J. Mahoney, \& A. Vazdekis (San Francisco, CA: ASP), 121

Cardelli, J. A., Clayton, G. C., \& Mathis, J. S. 1989, ApJ, 345, 245

Cox, A. L., Sparke, L. S., Watson, A. M., \& van Moorsel, G. 2001, AJ, 121, 692

Croxall, K. V., van Zee, L., Lee, H., Skillman, E. D., Lee, J. C., Côté, S., Kennicutt, R. C., \& Miller, B. W. 2009, ApJ, 705, 723

Dalcanton, J. J. 2007, ApJ, 658, 941

de Avillez, M. A., \& Mac Low, M. 2002, ApJ, 581, 1047

de Blok, W. J. G., \& van der Hulst, J. M. 1998, A\&A, 335, 421

Elson, E. C., de Blok, W. J. G., \& Kraan-Korteweg, R. C. 2010, MNRAS, 404, 2061

Ercolano, B., Bastian, N., \& Stasińska, G. 2007, MNRAS, 379, 945

Ferguson, A. M. N., \& Clarke, C. J. 2001, MNRAS, 325, 781

Ferguson, A. M. N., Gallagher, J. S., \& Wyse, R. F. G. 1998, AJ, 116, 673

Fumagalli, M., \& Gavazzi, G. 2008, A\&A, 490, 571

Hanish, D. J., et al. 2006, ApJ, 649, 150

Hibbard, J. E., van Gorkom, J. H., Rupen, M. P., \& Schiminovich, D. 2001, in ASP Conf. Ser. 240, Gas and Galaxy Evolution, ed. J. E. Hibbard, M. Rupen, \& J. H. van Gorkom (San Francisco, CA: ASP), 657

Hogg, D., \& Roberts, M. 2001, in ASP Conf. Ser. 240, Gas and Galaxy Evolution, ed. J. E. Hibbard, M. Rupen, \& J. H. van Gorkom (San Francisco, CA: ASP), 859
Huchtmeier, W. K., \& Richter, O. G. 1989, A General Catalog of HI Observations of Galaxies-the Reference Catalog (New York: Springer)

Hummer, D. G., \& Storey, P. J. 1987, MNRAS, 224, 801

Irwin, J. A. 1994, ApJ, 429, 618

Iyer, M., Simpson, C. E., Gottesman, S. T., Harsgis, J. R., \& Malphrus, B. K. 2001, in ASP Conf. Ser. 240, Gas and Galaxy Evolution, ed. J. E. Hibbard, M. Rupen, \& J. H. van Gorkom (San Francisco, CA: ASP), 861

Kahn, F. D. 1974, A\&A, 37, 149

Kennicutt, R. C., Jr., Bresolin, F., \& Garnett, D. R. 2003, ApJ, 591, 801

Kereš, D., Katz, N., Weinberg, D. H., \& Davé, R. 2005, MNRAS, 363, 2

Kewley, L. J., \& Ellison, S. L. 2008, ApJ, 681, 1183

Kewley, L. J., Rupke, D., Jabran Zahid, H., Geller, M. J., \& Barton, E. J. 2010, ApJ, 721, L48

Köppen, J., Weidner, C., \& Kroupa, P. 2007, MNRAS, 375, 673

Kregel, M., \& Sancisi, R. 2001, A\&A, 376, 59

Krisciunas, K., et al. 1987, PASP, 99, 887

Larson, R. B. 1974, MNRAS, 169, 229

Lee, J. C., et al. 2009, ApJ, 706, 599

Lequeux, J., Peimbert, M., Rayo, J. F., Serrano, A., \& Torres-Peimbert, S. 1979, A\&A, 80, 155

Leroy, A. K., Walter, F., Brinks, E., Bigiel, F., de Blok, W. J. G., Madore, B., \& Thornley, M. D. 2008, AJ, 136, 2782

Lisenfeld, U., Mundell, C. G., Schinnerer, E., Appleton, P. N., \& Allsopp, J. 2008, ApJ, 685, 181

Maeder, A. 1992, A\&A, 264, 105

Márquez, I., Masegosa, J., Moles, M., Varela, J., Bettoni, D., \& Galletta, G. 2002, A\&A, 393, 389

Martin, P., \& Roy, J. 1995, ApJ, 445, 161

McGaugh, S. S. 1991, ApJ, 380, 140

McNally, C. P., Wadsley, J., \& Couchman, H. M. P. 2009, ApJ, 697, L162

Meurer, G. R., et al. 2006, ApJS, 165, 307

Meurer, G. R., et al. 2009, ApJ, 695, 765

Minchev, I., \& Famaey, B. 2010, ApJ, 722, 112

Moustakas, J., \& Kennicutt, R. C., Jr. 2006, ApJS, 164, 81

Mundell, C. G. 2001, in ASP Conf. Ser. 240, Gas and Galaxy Evolution, ed. J. E. Hibbard, M. Rupen, \& J. H. van Gorkom (San Francisco, CA: ASP), 411

Oey, M. S. 2003, in IAU Symp. 212, A Massive Star Odyssey: From Main Sequence to Supernova, ed. K. van der Hucht, A. Herrero, \& C. Esteban (Cambridge: Cambridge Univ. Press), 620

Oey, M. S., \& Kennicutt, R. C., Jr. 1993, ApJ, 411, 137

Oppenheimer, B. D., \& Davé, R. 2009, MNRAS, 395, 1875

Osterbrock, D. E. (ed.) 1989, Astrophysics of Gaseous Nebulae and Active Galactic Nuclei (Mill Valley, CA: Univ. Science Books)

Pagel, B. E. J., Edmunds, M. G., Blackwell, D. E., Chun, M. S., \& Smith, G. 1979, MNRAS, 189, 95

Pettini, M., \& Pagel, B. E. J. 2004, MNRAS, 348, L59

Phillipps, S., \& Edmunds, M. G. 1991, MNRAS, 251, 84

Rupke, D. S. N., Kewley, L. J., \& Barnes, J. E. 2010, ApJ, 710, L156

Ryan-Weber, E., Webster, R., \& Bekki, K. 2003, in The IGM/Galaxy Connection, The Distribution of Baryons at $\mathrm{z}=0$, ed. J. L. Rosenberg \& $\mathrm{M}$ E. Putman (Astrophysics and Space Science Library, Vol. 281; Dordrecht: Kluwer), 223

Santillán, A., Sánchez-Salcedo, F. J., \& Franco, J. 2007, ApJ, 662, L19

Schaye, J. 2004, ApJ, 609, 667

Schlegel, D. J., Finkbeiner, D. P., \& Davis, M. 1998, ApJ, 500, 525

Searle, L. 1971, ApJ, 168, 327

Sellwood, J. A., \& Balbus, S. A. 1999, ApJ, 511, 660

Shi, F., Kong, X., Li, C., \& Cheng, F. Z. 2005, A\&A, 437, 849

Shields, G. A., \& Tinsley, B. M. 1976, ApJ, 203, 66

Skillman, E. D., Kennicutt, R. C., \& Hodge, P. W. 1989, ApJ, 347, 875

Smith, B. J. 1994, AJ, 107, 1695

Springob, C. M., Haynes, M. P., Giovanelli, R., \& Kent, B. R. 2005, ApJS, 160 149

Stasińska, G., \& Leitherer, C. 1996, ApJS, 107, 661

Strickland, D. K., Heckman, T. M., Colbert, E. J. M., Hoopes, C. G., \& Weaver, K. A. 2004, ApJ, 606, 829

Swaters, R. A., van Albada, T. S., van der Hulst, J. M., \& Sancisi, R. 2002, A\&A, 390,829

Tamburro, D., Rix, H., Leroy, A. K., Low, M., Walter, F., Kennicutt, R. C., Brinks, E., \& de Blok, W. J. G. 2009, AJ, 137, 4424

Taramopoulos, A., Payne, H., \& Briggs, F. H. 2001, A\&A, 365, 360

Tassis, K., Kravtsov, A. V., \& Gnedin, N. Y. 2008, ApJ, 672, 888

Tenorio-Tagle, G. 1996, AJ, 111, 1641

Tremonti, C. A., Moustakas, J., \& Diamond-Stanic, A. M. 2007, ApJ, 663, L77

Tremonti, C. A., et al. 2004, ApJ, 613, 898

van Zee, L. 2000, AJ, 119, 2757 
van Zee, L., \& Haynes, M. P. 2001, in ASP Conf. Ser. 240, Gas and Galaxy Evolution, ed. J. E. Hibbard, M. Rupen, \& J. H. van Gorkom (San Francisco, CA: ASP), 866

van Zee, L., \& Haynes, M. P. 2006, ApJ, 636, 214

Verheijen, M. A. W., \& Sancisi, R. 2001, A\&A, 370, 765

Vila-Costas, M. B., \& Edmunds, M. G. 1992, MNRAS, 259, 121

Wada, K., \& Norman, C. A. 1999, ApJ, 516, L13
Werk, J. K., Putman, M. E., Meurer, G. R., Thilker, D. A., Allen, R. J., BlandHawthorn, J., Kravtsov, A., \& Freeman, K. 2010a, ApJ, 715, 656

Werk, J. K., et al. 2010b, AJ, 139, 279

Wong, O. I., et al. 2006, MNRAS, 371, 1855

Wyder, T. K., et al. 2009, ApJ, 696, 1834

Zaritsky, D. 1992, ApJ, 390, L73

Zaritsky, D., Kennicutt, R. C., Jr., \& Huchra, J. P. 1994, ApJ, 420, 87 